\title{
Fractional Opial dynamic inequalities
}

\author{
A. G. Sayed ${ }^{a}$, S. H. Saker ${ }^{b, *}$, A. M. Ahmed ${ }^{a, c}$ \\ ${ }^{a}$ Department of Mathematics, Faculty of Science, Al-Azhar University, Nasr City 11884, Cairo, Egypt. \\ ${ }^{b}$ Department of Mathematics, Faculty of Science, Mansoura University, Mansoura 35516, Egypt. \\ ${ }^{c}$ Department of Mathematics, College of Science, Jouf University, Sakaka (2014), Kingdom of Saudi Arabia.
}

\begin{abstract}
In this paper, we will prove some new fractional dynamic inequalities on time scales of Opial's type. The results will be proved by employing the chain rule and Hölder's inequality on fractional time scales. As a special case of our results, when $\alpha=1$, we will obtain several well-known dynamic Opial inequalities on time scales.
\end{abstract}

Keywords: Opial's inequality, Hölder's inequality, time scales, conformable fractinonal calculus.

2020 MSC: 26A15, 26D10, 26D15, 39A13, 34A40, 34N05.

(C)2021 All rights reserved.

\section{Introduction}

In 2001, Bohner and Kaymakçalan [9] proved some dynamic inequalities of Opial type on time scales. One of the inequalities proved in [9] is given by

$$
\int_{0}^{a}\left|y(t)+y^{\sigma}(t)\right|\left|y^{\Delta}(t)\right| \Delta t \leqslant a \int_{0}^{a}\left|y^{\Delta}(t)\right|^{2} \Delta t
$$

where $y:[0, a] \cap \mathbb{T} \rightarrow \mathbb{R}$ is delta differentiable with $y(0)=0$. Also Bohner and Kaymakçalan in [9] proved that if $p$ and $q$ are positive functions on $[0, b]_{\mathbb{T}}, \int_{0}^{b}(\Delta t / p(t))<\infty, q$ is non-increasing, and $y:[0, b] \cap \mathbb{T} \rightarrow \mathbb{R}$ is delta differentiable with $y(0)=0$, then

$$
\int_{0}^{b} q^{\sigma}(t)\left|\left(y(t)+y^{\sigma}(t)\right) y^{\Delta}(t)\right| \Delta t \leqslant \int_{0}^{b} \frac{\Delta t}{p(t)} \int_{0}^{b} p(t) q(t)\left|y^{\Delta}(t)\right|^{2} \Delta t
$$

Karpuz et al. [17] replaced $q^{\sigma}(t)$ with $q(t)$ and proved an inequality similar to (1.1) of the form

$$
\int_{a}^{b} q(t)\left|\left(y(t)+y^{\sigma}(t)\right) y^{\Delta}(t)\right| \Delta t \leqslant K_{q}(a, b) \int_{a}^{b}\left|y^{\Delta}(t)\right|^{2} \Delta t
$$

\footnotetext{
*Corresponding author

Email addresses: a.g_sayed@yahoo.com (A. G. Sayed), shsaker@mans.edu.eg (S. H. Saker), ahmedelkb@yahoo.com \& amaahmed@ju.edu.sa (A. M. Ahmed)
}

doi: $10.22436 /$ jmcs.022.04.05

Received: 2020-06-22 Revised: 2020-07-22 Accepted: 2020-07-24 
where $q$ is a positive function on $[a, b]_{\mathbb{T}}, y:[a, b] \cap \mathbb{T} \rightarrow \mathbb{R}$ is delta differentiable with $y(0)=0$, and

$$
K_{q}(a, b)=\left(2 \int_{a}^{b} q^{2}(u)(\sigma(u)-a) \Delta u\right)^{1 / 2}
$$

Wong et al. [33] and Srivastava et al. [31] proved that if $\mathrm{q}$ is a positive and non-increasing function on $[a, b] \cap \mathbb{T}$, then

$$
\int_{a}^{b} q(t)|y(t)|^{\lambda}\left|y^{\Delta}(t)\right|^{\gamma} \Delta t \leqslant \frac{\gamma}{\lambda+\gamma}(b-a)^{\lambda} \int_{a}^{b} q(t)\left|y^{\Delta}(t)\right|^{\lambda+\gamma} \Delta t,
$$

where $y:[a, b] \cap \mathbb{T} \rightarrow \mathbb{R}$ is delta differentiable with $y(a)=0$. Saker [24] generalized (1.2) and proved some new dynamic inequalities with two weighted functions $p$ and $q$. In particular, he proved that if $p$ and $\mathrm{q}$ are nonnegative functions on $[\mathrm{a}, \mathrm{b}]_{\mathbb{T}}$ such that

$$
\int_{a}^{b} p^{-1 /(\lambda+\gamma-1)}(t) \Delta t<\infty,
$$

and $y:[a, b] \cap \mathbb{T} \rightarrow \mathbb{R}$ is delta differentiable with $y(a)=0$, then

$$
\int_{a}^{b} q(t)|y(t)|^{\lambda}\left|y^{\Delta}(t)\right|^{\gamma} \Delta t \leqslant K_{1}(a, b, \lambda, \gamma) \int_{a}^{b} p(t)\left|y^{\Delta}(t)\right|^{\lambda+\gamma} \Delta t
$$

where

$$
\mathrm{K}_{1}(\mathrm{a}, \mathrm{b}, \lambda, \gamma)=\left(\frac{\gamma}{\lambda+\gamma}\right)^{\frac{\gamma}{\lambda+\gamma}}\left(\int_{\mathrm{a}}^{\mathrm{b}} \frac{(\mathrm{q}(\mathrm{t}))^{(\lambda+\gamma) / \lambda}}{(\mathrm{p}(\mathrm{t}))^{\gamma / \lambda}}\left(\int_{\mathrm{a}}^{\mathrm{t}} \mathrm{p}^{\frac{-1}{(\lambda+\gamma-1)}}(\mathrm{s}) \Delta \mathrm{s}\right)^{(\lambda+\gamma-1)} \Delta \mathrm{t}\right)^{\frac{\lambda}{\lambda+\gamma}}
$$

In recent years, some authors studied the fractional inequalities by using the fractional Caputo and Riemann-Liouville derivative, we refer to the papers [7, 15, 34] for these results. In [1, 18] the authors extended the calculus of fractional order to conformable calculus. Recently, some authors have extended classical inequalities by using conformable fractional calculus such as Opial's inequality [27, 28], HermiteHadamard's inequality [13, 19], Chebyshev's inequality [5], and Steffensen's inequality [29].

Our aim in this paper is prove some new fractional dynamic inequalities of Opial's type on time scales which contain the classical Opial dynamic inequalities proved in [24]. The paper is divided into two sections. Section 2 is an introduction of basics of fractional calculus on time scales, and Section 3 contains the main results.

\section{Preliminaries and basic lemmas}

In this section, we present the basics of fractional calculus on time scales that will be needed throughout the paper. The results are adapted from $[6,11,12,21]$. A time scale $\mathbb{T}$ is an arbitrary nonempty closed subset of the real numbers $\mathbb{R}$. We assume throughout that $\mathbb{T}$ has the topology that it inherits from the standard topology on $\mathbb{R}$. We define the forward jump operator $\sigma: \mathbb{T} \rightarrow \mathbb{T}$, as

$$
\sigma(t):=\inf \{s \in \mathbb{T}: s>t\},
$$

while the backward jump operator $\rho: \mathbb{T} \rightarrow \mathbb{T}$, is defined by:

$$
\rho(t):=\sup \{s \in \mathbb{T}: s<t\},
$$

where $\sup \Phi=\inf \mathbb{T}$, and $\inf \Phi=\sup \mathbb{T}$ ( $\Phi$ is the empty set), for any $t \in \mathbb{T}$ the notation $f^{\sigma}(t)$ refers to $f(\sigma(t))$, i.e., $f^{\sigma}=f \circ \sigma$. Finally, the graininess function $\mu: \mathbb{T} \rightarrow[0, \infty)$ is defined by

$$
\mu(t):=\sigma(t)-t .
$$


Definition 2.1 (Conformable $\alpha$-fractional derivative). Let the function $f: \mathbb{T} \rightarrow \mathbb{R}$ and $\alpha \in(0,1]$. Then, for $t>0$, we define $T_{\alpha}^{\Delta} f(t)$ to be the number (provided it exists) with the property that, given any $\epsilon>0$, there is a neighborhood $U$ of $t$ such that for all $t \in U$,

$$
\left.\left|\left[f^{\sigma}(t)-f(s)\right] t^{1-\alpha}-T_{\alpha}^{\Delta} f(t)(\sigma(t)-s)\right| \leqslant \epsilon \mid \sigma(t)-s\right) \mid .
$$

$T_{\alpha}^{\Delta}(f(t))$ is called the conformable $\alpha$-fractional derivative of $f$ of order $\alpha$ at $t$ on $\mathbb{T}$, and we define the conformable fractional derivative on $\mathbb{T}$ at 0 as

$$
\mathrm{T}_{\alpha}^{\Delta} \mathrm{f}(0)=\lim _{\mathrm{t} \rightarrow 0} \mathrm{~T}_{\alpha}^{\Delta} \mathrm{f}(\mathrm{t})
$$

The conformable fractional derivative has the following properties.

Theorem 2.2. Let $u, v: \mathbb{T} \rightarrow \mathbb{R}$ be conformable fractional derivative of order $\alpha \in(0,1]$. Then the following properties are hold:

(i) the $u+v: \mathbb{T} \rightarrow \mathbb{R}$ is conformable fractional derivative and

$$
\mathrm{T}_{\alpha}^{\Delta}(\mathrm{u}+v)=\mathrm{T}_{\alpha}^{\Delta}(\mathrm{u})+\mathrm{T}_{\alpha}^{\Delta}(v) ;
$$

(ii) for any $\lambda \in \mathbb{R}$, then $\lambda u: \mathbb{T} \rightarrow \mathbb{R}$ is $\alpha$-fractional differentiable and

$$
\mathrm{T}_{\alpha}^{\Delta}(\lambda \mathrm{u})=\lambda \mathrm{T}_{\alpha}^{\Delta}(\mathrm{u})
$$

(iii) if $\mathrm{u}$ and $v$ are $\alpha$-fractional differentiable, then the product $\mathrm{u} v: \mathbb{T} \rightarrow \mathbb{R}$ is $\alpha$-fractional differentiable and

$$
\mathrm{T}_{\alpha}^{\Delta}(\mathrm{u} v)=\mathrm{T}_{\alpha}^{\Delta}(\mathrm{u}) v+(\mathrm{u} \circ \sigma) \mathrm{T}_{\alpha}^{\Delta}(v)=\mathrm{T}_{\alpha}^{\Delta}(\mathrm{u})(v \circ \sigma)+\mathrm{TT}_{\alpha}^{\Delta}(v)
$$

(iv) if $u$ is $\alpha$-fractional differentiable, then $1 / u$ is $\alpha$-fractional differentiable with

$$
\mathrm{T}_{\alpha}^{\Delta}\left(\frac{1}{\mathrm{u}}\right)=-\frac{\mathrm{T}_{\alpha}^{\Delta}(\mathrm{u})}{\mathrm{u}(\mathrm{u} \circ \sigma)}
$$

(v) if $\mathrm{u}$ and $v$ are $\alpha$-fractional differentiable, then $\mathrm{u} / \nu$ is $\alpha$-fractional differentiable with

$$
\mathrm{T}_{\alpha}^{\Delta}(\mathrm{u} / v)=\frac{\mathrm{T}_{\alpha}^{\Delta}(\mathrm{u}) v-\mathrm{u} \mathrm{T}_{\alpha}^{\Delta}(v)}{v(v \circ \sigma)}
$$

valid at all points $\mathrm{t} \in \mathbb{T}^{\mathrm{k}}$ for which $v(\mathrm{t})(v(\sigma(\mathrm{t})) \neq 0$.

Lemma 2.3 (Chain rule A). Let $v: \mathbb{T} \rightarrow \mathbb{R}$ be continuous and $\alpha$-fractional differentiable at $\mathrm{t} \in \mathbb{T}$, for $\alpha \in(0,1]$, and $\mathrm{u}: \mathbb{R} \rightarrow \mathbb{R}$ be continuously differentiable. Then there exists $\mathrm{d} \in[\mathrm{t}, \sigma(\mathrm{t})]$ with

$$
\mathrm{T}_{\alpha}^{\Delta}(\mathrm{u} \circ v)(\mathrm{t})=\mathrm{u}^{\prime}(v(\mathrm{~d})) \mathrm{T}_{\alpha}^{\Delta}(v(\mathrm{t})) .
$$

Lemma 2.4 (Chain rule B). Let $u: \mathbb{R} \rightarrow \mathbb{R}$ be continuously differentiable, and $v: \mathbb{T} \rightarrow \mathbb{R}$ be $\alpha$-fractional differentiable for $\alpha \in(0,1]$. Then $(u \circ v): \mathbb{T} \rightarrow \mathbb{R}$ is also $\alpha$-fractional differentiable, and we have

$$
\mathrm{T}_{\alpha}^{\Delta}(u \circ v)(s)=\left(\int_{0}^{1} u^{\prime}\left(v(s)+h \mu(s) s^{\alpha-1} \mathrm{~T}_{\alpha}^{\Delta}(v(s))\right) d h\right) \mathrm{T}_{\alpha}^{\Delta}(v(s)) .
$$

Definition 2.5 (Conformable fractional integral ). For $0<\alpha \leqslant 1$, then the $\alpha$-fractional integral of $f$, is defined by

$$
\int f(s) \Delta_{\alpha} s=\int f(s) s^{\alpha-1} \Delta s
$$


The conformable fractional integral satisfies the following properties.

Theorem 2.6. Let $\mathrm{a}, \mathrm{b}, \mathrm{c} \in \mathbb{T}, \lambda \in \mathbb{R}$ and let $\mathrm{u}, v: \mathbb{T} \rightarrow \mathbb{R}$. Then

(i) $\int_{a}^{b}[u(s)+v(s)] \Delta_{\alpha} s=\int_{a}^{b} u(s) \Delta_{\alpha} s+\int_{a}^{b} v(s) \Delta_{\alpha} s$;

(ii) $\int_{a}^{b} \lambda u(s) \Delta_{\alpha} s=\lambda \int_{a}^{b} u(s) \Delta_{\alpha} s$;

(iii) $\int_{a}^{b} u(s) \Delta_{\alpha} s=-\int_{b}^{a} u(s) \Delta_{\alpha} s$;

(iv) $\int_{a}^{b} u(s) \Delta_{\alpha} s=\int_{a}^{c} u(s) \Delta_{\alpha} s+\int_{c}^{b} u(s) \Delta_{\alpha} s$;

(v) $\int_{a}^{a} u(s) \Delta_{\alpha} s=0$.

Lemma 2.7 (Hölder's inequality). Let $\mathbb{T}$ be time scale, $\mathrm{a}, \mathrm{b} \in \mathbb{T}$ and $\alpha \in(0,1]$ and let $\mathrm{u}, v: \mathbb{T} \rightarrow \mathbb{R}$. Then

$$
\int_{a}^{b}|u(s) v(s)| \Delta_{\alpha} s \leqslant\left[\int_{a}^{b}|u(s)|^{p} \Delta_{\alpha} s\right]^{\frac{1}{p}}\left[\int_{a}^{b}|v(s)|^{q} \Delta_{\alpha} s\right]^{\frac{1}{q}}
$$

where $p>1$ and $1 / p+1 / q=1$.

\section{Main results}

In this section, we will prove our main results, the basic idea in proving the results is by using Hölder's inequality, chain rule for fractional on time scales. Throughout this paper (without mentioning it) we assume that the functions $\alpha$-fractional differentiable and integrals in the statements of the theorems are assumed to exist.

Theorem 3.1. Let $\mathbb{T}$ be a time scale with $\mathrm{a}, \tau \in \mathbb{T}, \gamma>1$, and $\mathrm{r}$ be a nonnegative nonincreasing on $[\mathrm{a}, \tau]_{\mathbb{T}}$. If $\mathrm{g}:[\mathrm{a}, \tau]_{\mathbb{T}} \rightarrow \mathbb{R}$ with $\mathrm{g}(\mathrm{a})=0$, and $\alpha \in(0,1]$, then for $\lambda \geqslant 0$

$$
\int_{a}^{\tau} r(t)|g(t)|^{\lambda}\left|T_{\alpha}^{\Delta} g(t)\right|^{\gamma} \Delta_{\alpha} t \leqslant\left(\frac{\gamma}{\lambda+\gamma}\right)\left(\frac{\tau^{\alpha}-a^{\alpha}}{\alpha}\right)^{\lambda} \int_{a}^{\tau} r(t)\left|T_{\alpha}^{\Delta} g(t)\right|^{\lambda+\gamma} \Delta_{\alpha} t .
$$

Proof. Let $u(t)=\int_{a}^{t} r^{\frac{\gamma}{\lambda+\gamma}}(s)\left|T_{\alpha}^{\Delta} g(s)\right|^{\gamma} \Delta_{\alpha} s$, for $t \in[a, \tau]_{\mathbb{T}}$. Then $u(a)=0$ and

$$
\mathrm{T}_{\alpha}^{\Delta} \mathrm{u}(\mathrm{t})=\mathrm{r}^{\frac{\gamma}{\lambda+\gamma}}(\mathrm{t})\left|\mathrm{T}_{\alpha}^{\Delta} \mathrm{g}(\mathrm{t})\right|^{\gamma}>0 .
$$

Applying Hölder's inequality (2.2) with indices $\gamma$ and $\gamma /(\gamma-1)$, so that

$$
\begin{aligned}
|g(t)|=\left|\int_{a}^{t} T_{\alpha}^{\Delta} g(s) \Delta_{\alpha} s\right| & \leqslant \int_{a}^{t}\left|T_{\alpha}^{\Delta} g(s)\right| \Delta_{\alpha} s \\
& =\int_{a}^{t} r^{\frac{-1}{\lambda+\gamma}}(s) r^{\frac{1}{\lambda+\gamma}}(s)\left|T_{\alpha}^{\Delta} g(s)\right| \Delta_{\alpha} s \\
& \leqslant\left(\int_{a}^{t}\left(r^{\frac{-1}{\lambda+\gamma}}(s)\right)^{\frac{\gamma}{\gamma-1}} \Delta_{\alpha} s\right)^{\frac{\gamma-1}{\gamma}}\left(\int_{a}^{t} r^{\frac{\gamma}{\lambda+\gamma}}(s)\left|T_{\alpha}^{\Delta} g(s)\right|^{\gamma} \Delta_{\alpha} s\right)^{\frac{1}{\gamma}} \\
& \leqslant r^{\frac{-1}{\lambda+\gamma}}(t)\left(\int_{a}^{t} \Delta_{\alpha} s\right)^{\frac{\gamma-1}{\gamma}}\left(\int_{a}^{t} r^{\frac{\gamma}{\lambda+\gamma}}(s)\left|T_{\alpha}^{\Delta} g(s)\right|^{\gamma} \Delta_{\alpha} s\right)^{\frac{1}{\gamma}} .
\end{aligned}
$$

Since $T_{\alpha}^{\Delta}\left(\frac{s^{\alpha}}{\alpha}\right) \geqslant 1$, this implies that

$$
\int_{a}^{t} \Delta_{\alpha} s \leqslant \int_{a}^{t} T_{\alpha}^{\Delta}\left(\frac{s^{\alpha}}{\alpha}\right) \Delta_{\alpha} s=\left.\frac{s^{\alpha}}{\alpha}\right|_{a} ^{t}=\frac{t^{\alpha}-a^{\alpha}}{\alpha} .
$$


Substituting (3.4) into (3.3), we see that

$$
|g(t)| \leqslant\left(\int_{a}^{t}\left(r^{\frac{-1}{\lambda+\gamma}}(s)\right)^{\frac{\gamma}{\gamma-1}} \Delta_{\alpha} s\right)^{\frac{\gamma-1}{\gamma}}\left(\int_{a}^{t} r^{\frac{\gamma}{\lambda+\gamma}}(s)\left|T_{\alpha}^{\Delta} g(s)\right|^{\gamma} \Delta_{\alpha} s\right)^{\frac{1}{\gamma}} \leqslant r^{\frac{-1}{\lambda+\gamma}}(t)\left(\frac{t^{\alpha}-a^{\alpha}}{\alpha}\right)^{\frac{\gamma-1}{\gamma}} u^{\frac{1}{\gamma}}(t),
$$

which yields that

$$
r^{\frac{\lambda}{\lambda+\gamma}}(s)|g(t)|^{\lambda} \leqslant\left(\frac{t^{\alpha}-a^{\alpha}}{\alpha}\right)^{\frac{\lambda(\gamma-1)}{\gamma}} u^{\frac{\lambda}{\gamma}}(t) .
$$

By applying the chain rule (2.1), we obtain

$$
\mathrm{T}_{\alpha}^{\Delta}\left(\mathrm{u}^{\frac{\lambda+\gamma}{\gamma}}(\mathrm{t})\right)=\frac{\lambda+\gamma}{\gamma} \mathrm{u}^{\frac{\lambda}{\gamma}}(\mathrm{d}) \mathrm{T}_{\alpha}^{\Delta} \mathrm{u}(\mathrm{t}), \text { where } \mathrm{d} \in[\mathrm{t}, \sigma(\mathrm{t})]
$$

Since $T_{\alpha}^{\Delta} \mathfrak{u}(t)=r^{\frac{\gamma}{\lambda+\gamma}}(t)\left|T_{\alpha}^{\Delta} g(t)\right|^{\gamma}>0$, and $d \geqslant t$, we see that

$$
\mathrm{T}_{\alpha}^{\Delta}\left(\mathrm{u}^{\frac{\lambda+\gamma}{\gamma}}(\mathrm{t})\right) \geqslant \frac{\lambda+\gamma}{\gamma} \mathrm{u}^{\frac{\lambda}{\gamma}}(\mathrm{t}) \mathrm{T}_{\alpha}^{\Delta} \mathrm{u}(\mathrm{t})
$$

Now, from (3.2), (3.5), and (3.6), we have that

$$
\begin{aligned}
\int_{a}^{\tau} r(t)|g(t)|^{\lambda}\left|T_{\alpha}^{\Delta} g(t)\right|^{\gamma} \Delta_{\alpha} t & =\int_{a}^{\tau} r^{\frac{\lambda}{\lambda+\gamma}}(t)|g(t)|^{\lambda} r^{\frac{\gamma}{\lambda+\gamma}}(t)\left|T_{\alpha}^{\Delta} g(t)\right|^{\gamma} \Delta_{\alpha} t \\
& \leqslant \int_{a}^{\tau}\left(\frac{t^{\alpha}-a^{\alpha}}{\alpha}\right)^{\frac{\lambda(\gamma-1)}{\gamma}} u^{\frac{\lambda}{\gamma}}(t) T_{\alpha}^{\Delta} u(t) \Delta_{\alpha} t \\
& \leqslant\left(\frac{\tau^{\alpha}-a^{\alpha}}{\alpha}\right)^{\frac{\lambda(\gamma-1)}{\gamma}} \int_{a}^{\tau} u^{\frac{\lambda}{\gamma}(t) T_{\alpha}^{\Delta}} u(t) \Delta_{\alpha} t \\
& \leqslant\left(\frac{\gamma}{\lambda+\gamma}\right)\left(\frac{\tau^{\alpha}-a^{\alpha}}{\alpha}\right)^{\frac{\lambda(\gamma-1)}{\gamma}} \int_{a}^{\tau} T_{\alpha}^{\Delta}\left(u^{\frac{\lambda+\gamma}{\gamma}}(t)\right) \Delta_{\alpha} t \\
& =\left(\frac{\gamma}{\lambda+\gamma}\right)\left(\frac{\tau^{\alpha}-a^{\alpha}}{\alpha}\right)^{\frac{\lambda(\gamma-1)}{\gamma}} u^{\frac{\lambda+\gamma}{\gamma}}(\tau) .
\end{aligned}
$$

By applying Hölder's inequality (2.2) with indices $(\lambda+\gamma) / \lambda$ and $(\lambda+\gamma) / \gamma$, and (3.4), we see that

$$
\begin{aligned}
u(\tau) & =\int_{a}^{\tau} r^{\frac{\gamma}{\lambda+\gamma}}(t)\left|T_{\alpha}^{\Delta} g(t)\right|^{\gamma} \Delta_{\alpha} t \\
& \leqslant\left(\int_{a}^{\tau} \Delta_{\alpha} t\right)^{\frac{\lambda}{\lambda+\gamma}}\left(\int_{a}^{\tau}\left(r^{\frac{\gamma}{\lambda+\gamma}}(t)\left|T_{\alpha}^{\Delta} g(t)\right|^{\gamma}\right)^{\frac{\lambda+\gamma}{\gamma}} \Delta_{\alpha} t\right)^{\frac{\gamma}{\lambda+\gamma}} \\
& \leqslant\left(\frac{\tau^{\alpha}-a^{\alpha}}{\alpha}\right)^{\frac{\lambda}{\lambda+\gamma}}\left(\int_{a}^{\tau} r(t)\left|T_{\alpha}^{\Delta} g(t)\right|^{\lambda+\gamma} \Delta_{\alpha} t\right)^{\frac{\gamma}{\lambda+\gamma}} .
\end{aligned}
$$

From (3.7) and (3.8), we have

$$
\int_{a}^{\tau} r(t)|g(t)|^{\lambda}\left|T_{\alpha}^{\Delta} g(t)\right|^{\gamma} \Delta_{\alpha} t \leqslant\left(\frac{\gamma}{\lambda+\gamma}\right)\left(\frac{\tau^{\alpha}-a^{\alpha}}{\alpha}\right)^{\lambda} \int_{a}^{\tau} r(t)\left|T_{\alpha}^{\Delta} g(t)\right|^{\lambda+\gamma} \Delta_{\alpha} t,
$$

which is the desired inequality (3.1). The proof is complete. 
Remark 3.2. As a special case of Theorem 3.1, when $\alpha=1$, then $\mathrm{T}_{\alpha}^{\Delta} \mathrm{g}(\mathrm{t})=\mathrm{g}^{\Delta}(\mathrm{t})$, and we obtain the inequality

which is the inequality (1.2).

$$
\int_{a}^{\tau} r(t)|g(t)|^{\lambda}\left|g^{\Delta}(t)\right|^{\gamma} \Delta t \leqslant \frac{\gamma(\tau-a)^{\lambda}}{\lambda+\gamma} \int_{a}^{\tau} r(t)\left|g^{\Delta}(t)\right|^{\lambda+\gamma} \Delta t
$$

Theorem 3.3. Let $\mathbb{T}$ be a time scale with $\mathrm{a}, \tau \in \mathbb{T}$, and $\mathrm{r} \in \mathrm{C}_{\mathrm{rd}}\left([\mathrm{a}, \tau]_{\mathbb{T}}, \mathbb{R}^{+}\right)$and $\mathrm{r}$ be a nonnegative and nonincreasing on $[a, \tau]_{\mathbb{T}}$. If $\mathrm{g}:[\mathrm{a}, \tau]_{\mathbb{T}} \rightarrow \mathbb{R}$ with $\mathrm{g}(\mathrm{a})=0$, and $\alpha \in(0,1]$, then for $\lambda \geqslant 0$

$$
\int_{a}^{\tau} r(t)|g(t)|^{\lambda}\left|T_{\alpha}^{\Delta} g(t)\right| \Delta_{\alpha} t \leqslant\left(\frac{1}{\lambda+1}\right)\left(\frac{\tau^{\alpha}-a^{\alpha}}{\alpha}\right)^{\lambda} \int_{a}^{\tau} r(t)\left|T_{\alpha}^{\Delta} g(t)\right|^{\lambda+1} \Delta_{\alpha} t .
$$

Proof. Let $u(t)=\int_{\alpha}^{t} r^{\frac{1}{\lambda+1}}(s)\left|T_{\alpha}^{\Delta} g(s)\right| \Delta_{\alpha} s$, for $t \in[a, \tau]_{\mathbb{T}}$. Then $u(a)=0$ and

$$
\begin{aligned}
|g(t)| & \leqslant \int_{a}^{t}\left|T_{\alpha}^{\Delta} g(s)\right| \Delta_{\alpha} s \leqslant \int_{a}^{t} r^{\frac{-1}{\lambda+1}}(s) r^{\frac{1}{\lambda+1}}(s)\left|T_{\alpha}^{\Delta} g(s)\right| \Delta_{\alpha} s \\
& \leqslant r^{\frac{-1}{\lambda+1}}(t) \int_{a}^{t} r^{\frac{1}{\lambda+1}}(s)\left|T_{\alpha}^{\Delta} g(s)\right| \Delta_{\alpha} s \leqslant r^{\frac{-1}{\lambda+1}}(t) u(t) .
\end{aligned}
$$

By applying the chain rule (2.1), we obtain

$$
\mathrm{T}_{\alpha}^{\Delta}\left(\mathrm{u}^{\lambda+1}(\mathrm{t})\right)=(\lambda+1) \mathrm{u}^{\lambda}(\mathrm{d}) \mathrm{T}_{\alpha}^{\Delta} \mathrm{u}(\mathrm{t}), \text { where } \mathrm{d} \in[\mathrm{t}, \sigma(\mathrm{t})] .
$$

Since $T_{\alpha}^{\Delta} \mathfrak{u}(t)=r^{\frac{1}{\lambda+1}}(t)\left|T_{\alpha}^{\Delta} g(t)\right|>0$, and $d \geqslant t$, we see that

$$
\mathrm{T}_{\alpha}^{\Delta}\left(\mathrm{u}^{\lambda+1}(\mathrm{t})\right)=(\lambda+1) \mathrm{u}^{\lambda}(\mathrm{d}) \mathrm{T}_{\alpha}^{\Delta} \mathrm{u}(\mathrm{t}) \geqslant(\lambda+1) \mathrm{u}^{\lambda}(\mathrm{t}) \mathrm{T}_{\alpha}^{\Delta} \mathrm{u}(\mathrm{t}) .
$$

Now, from (3.10) and (3.11) we have that

$$
\begin{aligned}
\int_{a}^{\tau} r(t)|g(t)|^{\lambda}\left|T_{\alpha}^{\Delta} g(t)\right| \Delta_{\alpha} t & \leqslant \int_{a}^{\tau} r^{\frac{\lambda}{\lambda+1}}(t)|g(t)|^{\lambda} \frac{1}{\lambda+1}(t)\left|T_{\alpha}^{\Delta} g(t)\right| \Delta_{\alpha} t \\
& \leqslant \int_{a}^{\tau} u^{\lambda}(t) T_{\alpha}^{\Delta} u(t) \Delta_{\alpha} t \leqslant \frac{1}{\lambda+1} \int_{a}^{\tau} T_{\alpha}^{\Delta}\left(u^{\lambda+1}(t)\right) \Delta_{\alpha} t=\frac{1}{\lambda+1} u^{\lambda+1}(\tau) .
\end{aligned}
$$

By applying Hölder's inequality (2.2) with indices $(\lambda+1)$ and $(\lambda+1) / \lambda$, and (3.4), we see that

$$
\begin{aligned}
u(\tau) & =\int_{a}^{\tau} r^{\frac{1}{\lambda+1}}(t)\left|T_{\alpha}^{\Delta} g(t)\right| \Delta_{\alpha} t \\
& \leqslant\left(\int_{a}^{\tau} \Delta_{\alpha} t\right)^{\frac{\lambda}{\lambda+1}}\left(\int_{a}^{\tau}\left(r^{\frac{1}{\lambda+1}}(t)\left|T_{\alpha}^{\Delta} g(t)\right|\right)^{\lambda+1} \Delta_{\alpha} t\right)^{\frac{1}{\lambda+1}} \\
& \leqslant\left(\frac{\tau^{\alpha}-a^{\alpha}}{\alpha}\right)^{\frac{\lambda}{\lambda+1}}\left(\int_{a}^{\tau}\left(r^{\frac{1}{\lambda+1}}(t)\left|T_{\alpha}^{\Delta} g(t)\right|\right)^{\lambda+1} \Delta_{\alpha} t\right)^{\frac{1}{\lambda+1}} \\
& =\left(\frac{\tau^{\alpha}-a^{\alpha}}{\alpha}\right)^{\frac{\lambda}{\lambda+1}}\left(\int_{a}^{\tau} r(t)\left|T_{\alpha}^{\Delta} g(t)\right|^{\lambda+1} \Delta_{\alpha} t\right)^{\frac{1}{\lambda+1}} .
\end{aligned}
$$

From (3.12) and (3.13), we have

$$
\int_{a}^{\tau} r(t)|g(t)|^{\lambda}\left|T_{\alpha}^{\Delta} g(t)\right| \Delta_{\alpha} t \leqslant\left(\frac{1}{\lambda+1}\right)\left(\frac{\tau^{\alpha}-a^{\alpha}}{\alpha}\right)^{\lambda} \int_{a}^{\tau} r(t)\left|T_{\alpha}^{\Delta} g(t)\right|^{\lambda+1} \Delta_{\alpha} t,
$$

which is the desired inequality (3.9). The proof is complete. 
Remark 3.4. As a special case of Theorem 3.3, when $\alpha=1$, then $\mathrm{T}_{\alpha}^{\Delta} \mathrm{g}(\mathrm{t})=\mathrm{g}^{\Delta}(\mathrm{t})$, and we obtain the inequality

$$
\int_{a}^{\tau} r(t)|g(t)|^{\lambda}\left|g^{\Delta}(t)\right| \Delta t \leqslant\left(\frac{1}{\lambda+1}\right)(\tau-a)^{\lambda} \int_{a}^{\tau} r(t)\left|g^{\Delta}(t)\right|^{\lambda+1} \Delta t
$$

Note that when $\lambda=1$, then the inequality (3.14) becomes

$$
\int_{a}^{\tau} r(t)|g(t)|\left|g^{\Delta}(t)\right| \Delta t \leqslant \frac{(\tau-a)}{2} \int_{a}^{\tau} r(t)\left|g^{\Delta}(t)\right|^{2} \Delta t
$$

Theorem 3.5. Let $\mathbb{T}$ be a time scale with $\tau, \mathrm{b} \in \mathbb{T}, \gamma>1$, and $\mathrm{r}$ be nonnegative and nondecreasing on $[\tau, \mathrm{b}]_{\mathbb{T}}$. If $\mathrm{g}:[\tau, \mathrm{b}]_{\mathbb{T}} \rightarrow \mathbb{R}$ with $\mathrm{g}(\mathrm{b})=0$, and $\alpha \in(0,1]$, then for $\lambda \geqslant 0$

$$
\int_{\tau}^{b} r(t)\left|g^{\sigma}(t)\right|^{\lambda}\left|T_{\alpha}^{\Delta} g(t)\right|^{\gamma} \Delta_{\alpha} t \leqslant\left(\frac{\gamma}{\lambda+\gamma}\right)\left(\frac{b^{\alpha}-\tau^{\alpha}}{\alpha}\right)^{\lambda} \int_{\tau}^{b} r(t)\left|T_{\alpha}^{\Delta} g(t)\right|^{\lambda+\gamma} \Delta_{\alpha} t .
$$

Proof. Let $u(t)=\int_{t}^{b} r^{\frac{\gamma}{\lambda+\gamma}}(s)\left|T_{\alpha}^{\Delta} g(s)\right|^{\gamma} \Delta_{\alpha} s$, for $t \in[\tau, b]_{\mathbb{T}}$. Then $u(b)=0$ and

$$
\mathrm{T}_{\alpha}^{\Delta} \mathrm{u}(\mathrm{t})=-\mathrm{r}^{\frac{\gamma}{\lambda+\gamma}}(\mathrm{t})\left|\mathrm{T}_{\alpha}^{\Delta} \mathrm{g}(\mathrm{t})\right|^{\gamma}<0 .
$$

Applying Hölder's inequality (2.2) with indices $\gamma$ and $\gamma /(\gamma-1)$, and (3.4), we see that

$$
\begin{aligned}
|g(t)|=\left|\int_{t}^{b} T_{\alpha}^{\Delta} g(s) \Delta_{\alpha} s\right| & \leqslant \int_{t}^{b}\left|T_{\alpha}^{\Delta} g(s)\right| \Delta_{\alpha} s \\
& =\int_{t}^{b} r^{\frac{-1}{\lambda+\gamma}}(s) r^{\frac{1}{\lambda+\gamma}}(s)\left|T_{\alpha}^{\Delta} g(s)\right| \Delta_{\alpha} s \\
& \leqslant\left(\int_{t}^{b}\left(r^{\frac{-1}{\lambda+\gamma}}(s)\right)^{\frac{\gamma}{\gamma-1}} \Delta_{\alpha} s\right)^{\frac{\gamma-1}{\gamma}}\left(\int_{t}^{b} r^{\frac{\gamma}{\lambda+\gamma}}(s)\left|T_{\alpha}^{\Delta} g(s)\right|^{\gamma} \Delta_{\alpha} s\right)^{\frac{1}{\gamma}} \\
& \leqslant r^{\frac{-1}{\lambda+\gamma}}(t)\left(\int_{t}^{b} \Delta_{\alpha} s\right)^{\frac{\gamma-1}{\gamma}}\left(\int_{t}^{b} r^{\frac{\gamma}{\lambda+\gamma}}(s)\left|T_{\alpha}^{\Delta} g(s)\right|^{\gamma} \Delta_{\alpha} s\right)^{\frac{1}{\gamma}} \\
& \leqslant r^{\frac{-1}{\lambda+\gamma}}(t)\left(\frac{b^{\alpha}-t^{\alpha}}{\alpha}\right)^{\frac{\gamma-1}{\gamma}} u^{\frac{1}{\gamma}(t)}
\end{aligned}
$$

which yields that

$$
r^{\frac{\lambda}{\lambda+\gamma}}(t)|g(t)|^{\lambda} \leqslant\left(\frac{b^{\alpha}-t^{\alpha}}{\alpha}\right)^{\frac{\lambda(\gamma-1)}{\gamma}} u^{\frac{\lambda}{\gamma}}(t) .
$$

By applying the chain rule (2.1), we obtain

$$
\mathrm{T}_{\alpha}^{\Delta}\left(\mathrm{u}^{\frac{\lambda+\gamma}{\gamma}}(\mathrm{t})\right)=\frac{\lambda+\gamma}{\gamma} \mathrm{u}^{\frac{\lambda}{\gamma}}(\mathrm{d}) \mathrm{T}_{\alpha}^{\Delta} \mathrm{u}(\mathrm{t}), \text { where } \mathrm{d} \in[\mathrm{t}, \sigma(\mathrm{t})]
$$

Since $T_{\alpha}^{\Delta} \mathfrak{u}(t)=-r^{\frac{\gamma}{\lambda+\gamma}}(t)\left|T_{\alpha}^{\Delta} g(t)\right|^{\gamma}<0$, and $\sigma(t) \geqslant d$, this implies that

$$
-\mathfrak{u}^{\frac{\lambda}{\gamma}}(\sigma(t)) T_{\alpha}^{\Delta} \mathfrak{u}(t) \leqslant-u^{\frac{\lambda}{\gamma}}(d) T_{\alpha}^{\Delta} \mathfrak{u}(t) \leqslant-u^{\frac{\lambda}{\gamma}}(t) T_{\alpha}^{\Delta} \mathfrak{u}(t)
$$

and we see that

$$
-\mathrm{T}_{\alpha}^{\Delta}\left(\mathrm{u}^{\frac{\lambda+\gamma}{\gamma}}(\mathrm{t})\right) \geqslant \frac{\lambda+\gamma}{\gamma}\left(-\mathrm{u}^{\frac{\lambda}{\gamma}}(\sigma(\mathrm{t})) \mathrm{T}_{\alpha}^{\Delta} \mathrm{u}(\mathrm{t})\right) .
$$


Now, from (3.16), (3.17), and (3.18) we have that

$$
\begin{aligned}
\int_{\tau}^{b} r(t)\left|g^{\sigma}(t)\right|^{\lambda}\left|T_{\alpha}^{\Delta} g(t)\right|^{\gamma} \Delta_{\alpha} t & =\int_{\tau}^{b} r^{\frac{\lambda}{\lambda+\gamma}}(t)\left|g^{\sigma}(t)\right|^{\lambda} r^{\frac{\gamma}{\lambda+\gamma}}(t)\left|T_{\alpha}^{\Delta} g(t)\right|^{\gamma} \Delta_{\alpha} t \\
& \leqslant \int_{\tau}^{b} r^{\frac{\lambda}{\lambda+\gamma}}(\sigma(t))\left|g^{\sigma}(t)\right|^{\lambda} r^{\frac{\gamma}{\lambda+\gamma}}(t)\left|T_{\alpha}^{\Delta} g(t)\right|^{\gamma} \Delta_{\alpha} t \\
& \leqslant \int_{\tau}^{b}\left(\frac{b^{\alpha}-(\sigma(t))^{\alpha}}{\alpha}\right)^{\frac{\lambda(\gamma-1)}{\gamma}}\left(-u^{\frac{\lambda}{\gamma}}(\sigma(t)) T_{\alpha}^{\Delta} u(t)\right) \Delta_{\alpha} t \\
& \leqslant\left(\frac{b^{\alpha}-\tau^{\alpha}}{\alpha}\right)^{\frac{\lambda(\gamma-1)}{\gamma}} \int_{\tau}^{b}\left(-u^{\frac{\lambda}{\gamma}}(\sigma(t)) T_{\alpha}^{\Delta} u(t)\right) \Delta_{\alpha} t \\
& \leqslant \frac{\gamma}{\lambda+\gamma}\left(\frac{b^{\alpha}-\tau^{\alpha}}{\alpha}\right)^{\frac{\lambda(\gamma-1)}{\gamma}} \int_{\tau}^{b}-T_{\alpha}^{\Delta}\left(u^{\frac{\lambda+\gamma}{\gamma}}(t)\right) \Delta_{\alpha} t \\
& =\frac{\gamma}{\lambda+\gamma}\left(\frac{b^{\alpha}-\tau^{\alpha}}{\alpha}\right)^{\frac{\lambda(\gamma-1)}{\gamma}} u^{\frac{\lambda+\gamma}{\gamma}}(\tau) .
\end{aligned}
$$

By applying Hölder's inequality (2.2) with indices $(\lambda+\gamma) / \lambda$ and $(\lambda+\gamma) / \gamma$, and (3.4), we see that

$$
\begin{aligned}
u(\tau) & =\int_{\tau}^{b} r^{\frac{\gamma}{\lambda+\gamma}}(t)\left|T_{\alpha}^{\Delta} g(t)\right|^{\gamma} \Delta_{\alpha} t \\
& \leqslant\left(\int_{\tau}^{b} \Delta_{\alpha} t\right)^{\frac{\lambda}{\lambda+\gamma}}\left(\int_{\tau}^{b}\left(r^{\frac{\gamma}{\lambda+\gamma}}(t)\left|T_{\alpha}^{\Delta} g(t)\right|^{\gamma}\right)^{\frac{\lambda+\gamma}{\gamma}} \Delta_{\alpha} t\right)^{\frac{\gamma}{\lambda+\gamma}} \\
& \leqslant\left(\frac{b^{\alpha}-\tau^{\alpha}}{\alpha}\right)^{\frac{\lambda}{\lambda+\gamma}}\left(\int_{\tau}^{b} r(t)\left|T_{\alpha}^{\Delta} g(t)\right|^{\lambda+\gamma} \Delta_{\alpha} t\right)^{\frac{\gamma}{\lambda+\gamma}}
\end{aligned}
$$

From (3.19) and (3.20), we have

$$
\int_{\tau}^{b} r(t)\left|g^{\sigma}(t)\right|^{\lambda}\left|T_{\alpha}^{\Delta} g(t)\right|^{\gamma} \Delta_{\alpha} t \leqslant\left(\frac{\gamma}{\lambda+\gamma}\right)\left(\frac{b^{\alpha}-\tau^{\alpha}}{\alpha}\right)^{\lambda} \int_{\tau}^{b} r(t)\left|T_{\alpha}^{\Delta} g(t)\right|^{\lambda+\gamma} \Delta_{\alpha} t,
$$

which is the desired inequality (3.15). The proof is complete.

Remark 3.6. As a special case of Theorem 3.5, when $\alpha=1$, then $\mathrm{T}_{\alpha}^{\Delta} \mathrm{g}(\mathrm{t})=\mathrm{g}^{\Delta}(\mathrm{t})$, and we obtain the inequality

$$
\int_{\tau}^{b} r(t)\left|g^{\sigma}(t)\right|^{\lambda}\left|g^{\Delta}(t)\right|^{\gamma} \Delta t \leqslant \frac{\gamma(b-\tau)^{\lambda}}{\lambda+\gamma} \int_{\tau}^{b} r(t)\left|g^{\Delta}(t)\right|^{\lambda+\gamma} \Delta t .
$$

Theorem 3.7. Let $\mathbb{T}$ be a time scale with $\tau, \mathrm{b} \in \mathbb{T}$, and $\mathrm{r} \in \mathrm{C}_{\mathrm{rd}}\left([\tau, \mathrm{b}]_{\mathbb{T}}, \mathbb{R}^{+}\right)$be such that $\mathrm{r}$ is nondecreasing on $[\tau, \mathrm{b}]_{\mathbb{T}}$. If $\mathrm{g}:[\tau, \mathrm{b}]_{\mathbb{T}} \rightarrow \mathbb{R}$ with $\mathrm{g}(\mathrm{b})=0$, and $\alpha \in(0,1]$, then for $\lambda \geqslant 0$

$$
\int_{\tau}^{\mathrm{b}} r(\mathrm{t})\left|g^{\sigma}(\mathrm{t})\right|^{\lambda}\left|\mathrm{T}_{\alpha}^{\Delta} \mathrm{g}(\mathrm{t})\right| \Delta_{\alpha} \mathrm{t} \leqslant\left(\frac{1}{\lambda+1}\right)\left(\frac{\mathrm{b}^{\alpha}-\tau^{\alpha}}{\alpha}\right)^{\lambda} \int_{\tau}^{\mathrm{b}} r(\mathrm{t})\left|\mathrm{T}_{\alpha}^{\Delta} \mathrm{g}(\mathrm{t})\right|^{\lambda+1} \Delta_{\alpha} \mathrm{t} .
$$


Proof. Let $u(t)=\int_{t}^{b} r^{\frac{1}{\lambda+1}}(s)\left|T_{\alpha}^{\Delta} g(s)\right| \Delta_{\alpha} s$, for $t \in[\tau, b]_{\mathbb{T}}$. Then $u(b)=0$ and

$$
\begin{aligned}
|g(t)|=\left|\int_{t}^{b} T_{\alpha}^{\Delta} g(s) \Delta_{\alpha} s\right| & \leqslant \int_{t}^{b}\left|T_{\alpha}^{\Delta} g(s)\right| \Delta_{\alpha} s \\
& =\int_{t}^{b} r^{\frac{-1}{\lambda+1}}(s) r^{\frac{1}{\lambda+1}}(s)\left|T_{\alpha}^{\Delta} g(s)\right| \Delta_{\alpha} s \\
& \leqslant r^{\frac{-1}{\lambda+1}}(t) \int_{t}^{b} r^{\frac{1}{\lambda+1}}(s)\left|T_{\alpha}^{\Delta} g(s)\right| \Delta_{\alpha} s \leqslant r^{\frac{-1}{\lambda+1}}(t) u(t) .
\end{aligned}
$$

By applying the chain rule (2.1), we obtain

$$
\mathrm{T}_{\alpha}^{\Delta}\left(\mathrm{u}^{\lambda+1}(\mathrm{t})\right)=(\lambda+1) \mathrm{u}^{\lambda}(\mathrm{d}) \mathrm{T}_{\alpha}^{\Delta} \mathrm{u}(\mathrm{t}), \text { where } \mathrm{d} \in[\mathrm{t}, \sigma(\mathrm{t})] .
$$

Since $T_{\alpha}^{\Delta} \mathbf{u}(t)=-r^{\frac{1}{\lambda+1}}(t)\left|T_{\alpha}^{\Delta} g(t)\right|<0$, and $\sigma(t) \geqslant d$, this implies that

$$
-u^{\lambda}(\sigma(t)) T_{\alpha}^{\Delta} u(t) \leqslant-u^{\lambda}(d) T_{\alpha}^{\Delta} u(t) \leqslant-u^{\lambda}(t) T_{\alpha}^{\Delta} u(t)
$$

and we see that

$$
-T_{\alpha}^{\Delta}\left(u^{\lambda+1}(t)\right)=-(\lambda+1) u^{\lambda}(d) T_{\alpha}^{\Delta} u(t) \geqslant-(\lambda+1) u^{\lambda}(\sigma(t)) T_{\alpha}^{\Delta} u(t) .
$$

Now, from (3.22) and (3.23) we have that

$$
\begin{aligned}
\int_{\tau}^{\mathrm{b}} r(t)\left|g^{\sigma}(t)\right|^{\lambda}\left|T_{\alpha}^{\Delta} g(t)\right| \Delta_{\alpha} t & =\int_{\tau}^{b} r^{\frac{\lambda}{\lambda+1}}(t)\left|g^{\sigma}(t)\right|^{\lambda} r^{\frac{1}{\lambda+1}}(t)\left|T_{\alpha}^{\Delta} g(t)\right| \Delta_{\alpha} t \\
& \leqslant \int_{\tau}^{b} r \frac{\lambda}{\lambda+1}(\sigma(t))\left|g^{\sigma}(t)\right|^{\lambda} r^{\frac{1}{\lambda+1}}(t)\left|T_{\alpha}^{\Delta} g(t)\right| \Delta_{\alpha} t \\
& \leqslant-\int_{\tau}^{b} u^{\lambda}(\sigma(t)) T_{\alpha}^{\Delta} u(t) \Delta_{\alpha} t \\
& \leqslant-\int_{\tau}^{b} u^{\lambda}(d) T_{\alpha}^{\Delta} u(t) \Delta_{\alpha} t \\
& =\frac{1}{\lambda+1} \int_{\tau}^{b} T_{\alpha}^{\Delta}\left(-u^{\lambda+1}(t)\right) \Delta_{\alpha} t=\frac{1}{\lambda+1} u^{\lambda+1}(\tau)
\end{aligned}
$$

By applying Hölder's inequality (2.2) with indices $(\lambda+1)$ and $(\lambda+1) / \lambda$, and (3.4), we see that

$$
\begin{aligned}
u(\tau) & =\int_{\tau}^{b} r^{\frac{1}{\lambda+1}}(t)\left|T_{\alpha}^{\Delta} g(t)\right| \Delta_{\alpha} t \\
& \leqslant\left(\int_{\tau}^{b} \Delta_{\alpha} t\right)^{\frac{\lambda}{\lambda+1}}\left(\int_{\tau}^{b}\left(r^{\frac{1}{\lambda+1}}(t)\left|T_{\alpha}^{\Delta} g(t)\right|\right)^{\lambda+1} \Delta_{\alpha} t\right)^{\frac{1}{\lambda+1}} \\
& \leqslant\left(\frac{b^{\alpha}-\tau^{\alpha}}{\alpha}\right)^{\frac{\lambda}{\lambda+1}}\left(\int_{\tau}^{b} r(t)\left|T_{\alpha}^{\Delta} g(t)\right|^{\lambda+1} \Delta_{\alpha} t\right)^{\frac{1}{\lambda+1}}
\end{aligned}
$$


From (3.24) and (3.25) we have that

$$
\int_{\tau}^{b} r(t)\left|g^{\sigma}(t)\right|^{\lambda}\left|T_{\alpha}^{\Delta} g(t)\right| \Delta_{\alpha} t \leqslant \frac{1}{\lambda+1}\left(\frac{b^{\alpha}-\tau^{\alpha}}{\alpha}\right)^{\lambda} \int_{\tau}^{b} r(t)\left|T_{\alpha}^{\Delta} g(t)\right|^{\lambda+1} \Delta_{\alpha} t,
$$

which is the desired inequality (3.21). The proof is complete.

Remark 3.8. As a special case of Theorem 3.7, when $\alpha=1$, then $\mathrm{T}_{\alpha}^{\Delta} \mathrm{g}(\mathrm{t})=\mathrm{g}^{\Delta}(\mathrm{t})$, and we obtain the inequality

$$
\int_{\tau}^{b} r(t)\left|g^{\sigma}(t)\right|^{\lambda}\left|g^{\Delta}(t)\right| \Delta t \leqslant \frac{(b-\tau)^{\lambda}}{\lambda+1} \int_{\tau}^{b} r(t)\left|g^{\Delta}(t)\right|^{\lambda+1} \Delta t,
$$

note that when $\lambda=1$, then the inequality (3.26) becomes

$$
\int_{\tau}^{b} r(t)\left|g^{\sigma}(t)\right|\left|g^{\Delta}(t)\right| \Delta t \leqslant \frac{(b-\tau)}{2} \int_{\tau}^{b} r(t)\left|g^{\Delta}(t)\right|^{2} \Delta t .
$$

Theorem 3.9. Let $\mathbb{T}$ be a time scale with $a, \tau \in \mathbb{T}, \gamma>1$, and $s \in \mathrm{C}_{\mathrm{rd}}\left([\mathrm{a}, \tau]_{\mathbb{T}}, \mathbb{R}^{+}\right)$be such that $\mathrm{s}(\mathrm{t})$ is nonincreasing on $[a, \tau]_{\mathbb{T}}$, and let $r$ be a nonnegative function on $(a, \tau)_{\mathbb{T}}$ such that $\int_{a}^{\tau} r^{-1 /(\lambda+\gamma)}(t) \Delta_{\alpha} t<\infty$. If $\mathrm{g}:[\mathrm{a}, \tau]_{\mathbb{T}} \rightarrow \mathbb{R}$ with $\mathrm{g}(\mathrm{a})=0$, and $\alpha \in(0,1]$, then for $\lambda \geqslant 0$

$$
\int_{a}^{\tau} s(t)|g(t)|^{\lambda}\left|T_{\alpha}^{\Delta} g(t)\right|^{\gamma} \Delta_{\alpha} t \leqslant K_{1}(a, \tau, \lambda, \gamma) \int_{a}^{\tau} r(t)\left|T_{\alpha}^{\Delta} g(t)\right|^{\lambda+\gamma} \Delta_{\alpha} t
$$

where

$$
K_{1}(a, \tau, \lambda, \gamma)=\left(\frac{\gamma}{\lambda+\gamma}\right)\left(\frac{\tau^{\alpha}-a^{\alpha}}{\alpha}\right)^{\frac{\lambda(\gamma-1)}{\gamma}}\left(\int_{a}^{\tau}\left(\frac{s(t)}{r(t)}\right)^{\gamma / \lambda} \Delta_{\alpha} t\right)^{\lambda / \gamma}
$$

Proof. Let $u(t)=\int_{a}^{t} s^{\frac{\gamma}{\lambda+\gamma}}(x)\left|T_{\alpha}^{\Delta} g(x)\right|^{\gamma} \Delta_{\alpha} x$, for $t \in[a, \tau]_{\mathbb{T}}$. Then $u(a)=0$ and

$$
\mathrm{T}_{\alpha}^{\Delta} \mathrm{u}(\mathrm{t})=\mathrm{s}^{\frac{\gamma}{\lambda+\gamma}}(\mathrm{t})\left|\mathrm{T}_{\alpha}^{\Delta} \mathrm{g}(\mathrm{t})\right|^{\gamma}>0 .
$$

Applying Hölder's inequality (2.2) with indices $\gamma$ and $\gamma /(\gamma-1)$, so that

$$
\begin{aligned}
|g(t)|=\left|\int_{a}^{t} T_{\alpha}^{\Delta} g(x) \Delta_{\alpha} x\right| & \leqslant \int_{a}^{t}\left|T_{\alpha}^{\Delta} g(x)\right| \Delta_{\alpha} x \\
& =\int_{a}^{t} s^{\frac{-1}{\lambda+\gamma}}(x) s^{\frac{1}{\lambda+\gamma}}(x)\left|T_{\alpha}^{\Delta} g(x)\right| \Delta_{\alpha} x \\
& \leqslant\left(\int_{a}^{t}\left(s^{\frac{-1}{\lambda+\gamma}}(x)\right)^{\frac{\gamma}{\gamma-1}} \Delta_{\alpha} x\right)^{\frac{\gamma-1}{\gamma}}\left(\int_{a}^{t} s^{\frac{\gamma}{\lambda+\gamma}}(x)\left|T_{\alpha}^{\Delta} g(x)\right|^{\gamma} \Delta_{\alpha} x\right)^{\frac{1}{\gamma}} .
\end{aligned}
$$

Since $T_{\alpha}^{\Delta}\left(\frac{x^{\alpha}}{\alpha}\right) \geqslant 1$, this implies that

$$
\int_{a}^{t} \Delta_{\alpha} x \leqslant \int_{a}^{t} T_{\alpha}^{\Delta}\left(\frac{x^{\alpha}}{\alpha}\right) \Delta_{\alpha} x=\left.\frac{x^{\alpha}}{\alpha}\right|_{a} ^{t}=\frac{t^{\alpha}-a^{\alpha}}{\alpha} .
$$

Substituting (3.30) into (3.29), we see that

$$
|g(t)| \leqslant\left(\int_{a}^{t}\left(s^{\frac{-1}{\lambda+\gamma}}(x)\right)^{\frac{\gamma}{\gamma-1}} \Delta_{\alpha} x\right)^{\frac{\gamma-1}{\gamma}}\left(\int_{a}^{t} s^{\frac{\gamma}{\lambda+\gamma}}(x)\left|T_{\alpha}^{\Delta} g(x)\right|^{\gamma} \Delta_{\alpha} x\right)^{\frac{1}{\gamma}}
$$




$$
\leqslant s^{\frac{-1}{\lambda+\gamma}}(t)\left(\int_{a}^{t} \Delta_{\alpha} x\right)^{\frac{\gamma-1}{\gamma}}\left(\int_{a}^{t} s^{\frac{\gamma}{\lambda+\gamma}}(x)\left|T_{\alpha}^{\Delta} g(x)\right|^{\gamma} \Delta_{\alpha} x\right)^{\frac{1}{\gamma}} \leqslant s^{\frac{-1}{\lambda+\gamma}}(t)\left(\frac{t^{\alpha}-a^{\alpha}}{\alpha}\right)^{\frac{\gamma-1}{\gamma}} u^{\frac{1}{\gamma}}(t),
$$

which yields that

$$
\mathbf{s}^{\frac{\lambda}{\lambda+\gamma}}(t)|g(t)|^{\lambda} \leqslant\left(\frac{t^{\alpha}-a^{\alpha}}{\alpha}\right)^{\frac{\lambda(\gamma-1)}{\gamma}} u^{\frac{\lambda}{\gamma}}(t) .
$$

By applying the chain rule (2.1), we obtain

$$
\mathrm{T}_{\alpha}^{\Delta}\left(\mathrm{u}^{\frac{\lambda+\gamma}{\gamma}}(\mathrm{t})\right)=\frac{\lambda+\gamma}{\gamma} \mathrm{u}^{\frac{\lambda}{\gamma}}(\mathrm{d}) \mathrm{T}_{\alpha}^{\Delta} \mathrm{u}(\mathrm{t}), \text { where } \mathrm{d} \in[\mathrm{t}, \sigma(\mathrm{t})]
$$

Since $T_{\alpha}^{\Delta} u(t)=r^{\frac{\gamma}{\lambda+\gamma}}(t)\left|T_{\alpha}^{\Delta} g(t)\right|^{\gamma}>0$, and $d \geqslant t$, we see that

$$
\mathrm{T}_{\alpha}^{\Delta}\left(\mathrm{u}^{\frac{\lambda+\gamma}{\gamma}}(\mathrm{t})\right) \geqslant \frac{\lambda+\gamma}{\gamma} \mathrm{u}^{\frac{\lambda}{\gamma}}(\mathrm{t}) \mathrm{T}_{\alpha}^{\Delta} \mathrm{u}(\mathrm{t})
$$

Now, from (3.28), (3.31), and (3.32), we have that

$$
\begin{aligned}
\int_{a}^{\tau} s(t)|g(t)|^{\lambda}\left|T_{\alpha}^{\Delta} g(t)\right|^{\gamma} \Delta_{\alpha} t & =\int_{a}^{\tau} s^{\frac{\lambda}{\lambda+\gamma}}(t)|g(t)|^{\lambda} s^{\frac{\gamma}{\lambda+\gamma}}(t)\left|T_{\alpha}^{\Delta} g(t)\right|^{\gamma} \Delta_{\alpha} t \\
& \leqslant \int_{a}^{\tau}\left(\frac{t^{\alpha}-a^{\alpha}}{\alpha}\right)^{\frac{\lambda(\gamma-1)}{\gamma}} u^{\frac{\lambda}{\gamma}}(t) T_{\alpha}^{\Delta} u(t) \Delta_{\alpha} t \\
& \leqslant\left(\frac{\tau^{\alpha}-a^{\alpha}}{\alpha}\right)^{\frac{\lambda(\gamma-1)}{\gamma}} \int_{a}^{\tau} u^{\frac{\lambda}{\gamma}(t) T_{\alpha}^{\Delta}} u(t) \Delta_{\alpha} t \\
& \leqslant\left(\frac{\gamma}{\lambda+\gamma}\right)\left(\frac{\tau^{\alpha}-a^{\alpha}}{\alpha}\right)^{\frac{\lambda(\gamma-1)}{\gamma}} \int_{a}^{\tau} T_{\alpha}^{\Delta}\left(u^{\frac{\lambda+\gamma}{\gamma}}(t)\right) \Delta_{\alpha} t \\
& =\left(\frac{\gamma}{\lambda+\gamma}\right)\left(\frac{\tau^{\alpha}-a^{\alpha}}{\alpha}\right)^{\frac{\lambda(\gamma-1)}{\gamma}} u^{\frac{\lambda+\gamma}{\gamma}}(\tau) .
\end{aligned}
$$

By applying Hölder's inequality (2.2) with indices $(\lambda+\gamma) / \lambda$ and $(\lambda+\gamma) / \gamma$, and (3.30), we see that

$$
\begin{aligned}
\int_{a}^{\tau} s(t)|g(t)|^{\lambda}\left|T_{\alpha}^{\Delta} g(t)\right|^{\gamma} \Delta_{\alpha} t \leqslant & \left(\frac{\gamma}{\lambda+\gamma}\right)\left(\frac{\tau^{\alpha}-a^{\alpha}}{\alpha}\right)^{\frac{\lambda(\gamma-1)}{\gamma}} u^{\frac{\lambda+\gamma}{\gamma}}(\tau) \\
= & \left(\frac{\gamma}{\lambda+\gamma}\right)\left(\frac{\tau^{\alpha}-a^{\alpha}}{\alpha}\right)^{\frac{\lambda(\gamma-1)}{\gamma}}\left(\int_{a}^{\tau} s^{\frac{\gamma}{\lambda+\gamma}}(t) r^{\frac{-\gamma}{\lambda+\gamma}}(t)\right. \\
& \left.\times r^{\frac{\gamma}{\lambda+\gamma}}(t)\left|T_{\alpha}^{\Delta} g(t)\right|^{\gamma} \Delta_{\alpha} t\right)^{\frac{\lambda+\gamma}{\gamma}} \\
\leqslant & \left(\frac{\gamma}{\lambda+\gamma}\right)\left(\frac{\tau^{\alpha}-a^{\alpha}}{\alpha}\right)^{\frac{\lambda(\gamma-1)}{\gamma}}\left(\int_{a}^{\tau}\left(\frac{s(t)}{r(t)}\right)^{\gamma / \lambda} \Delta_{\alpha} t\right)^{\lambda / \gamma} \\
& \times \int_{a}^{\tau} r(t)\left|T_{\alpha}^{\Delta} g(t)\right|^{\lambda+\gamma} \Delta_{\alpha} t \\
= & K_{1}(a, \tau, \lambda, \gamma) \int_{a}^{\tau} r(t)\left|T_{\alpha}^{\Delta} g(t)\right|^{\lambda+\gamma} \Delta_{\alpha} t,
\end{aligned}
$$

where

$$
K_{1}(a, \tau, \lambda, \gamma)=\left(\frac{\gamma}{\lambda+\gamma}\right)\left(\frac{\tau^{\alpha}-a^{\alpha}}{\alpha}\right)^{\frac{\lambda(\gamma-1)}{\gamma}}\left(\int_{a}^{\tau}\left(\frac{s(t)}{r(t)}\right)^{\gamma / \lambda} \Delta_{\alpha} t\right)^{\lambda / \gamma},
$$

which is the desired inequality (3.27). The proof is complete. 
Remark 3.10. As a special case of Theorem 3.9, when $s(t)=r(t)$, then we get

$$
\int_{a}^{\tau} r(t)|g(t)|^{\lambda}\left|T_{\alpha}^{\Delta} g(t)\right|^{\gamma} \Delta_{\alpha} t \leqslant\left(\frac{\gamma}{\lambda+\gamma}\right)\left(\frac{\tau^{\alpha}-a^{\alpha}}{\alpha}\right)^{\lambda} \int_{a}^{\tau} r(t)\left|T_{\alpha}^{\Delta} g(t)\right|^{\lambda+\gamma} \Delta_{\alpha} t,
$$

and when $\alpha=1$, then $\mathrm{T}_{\alpha}^{\Delta} \mathrm{g}(\mathrm{t})=\mathrm{g}^{\Delta}(\mathrm{t})$, and we obtain the inequality

$$
\int_{a}^{\tau} r(t)|g(t)|^{\lambda}\left|g^{\Delta}(t)\right|^{\gamma} \Delta t \leqslant \frac{\gamma(\tau-a)^{\lambda}}{\lambda+\gamma} \int_{a}^{\tau} r(t)\left|g^{\Delta}(t)\right|^{\lambda+\gamma} \Delta t
$$

Theorem 3.11. Let $\mathbb{T}$ be a time scale with $\mathrm{a}, \tau \in \mathbb{T}$, and $\mathrm{s} \in \mathrm{C}_{\mathrm{rd}}\left([\mathrm{a}, \tau]_{\mathbb{T}}, \mathbb{R}^{+}\right)$be such that $\mathrm{s}(\mathrm{t})$ is nonincreasing on $[a, \tau]_{\mathbb{T}}$, and let $r$ be a nonnegative function on $(a, \tau)_{\mathbb{T}}$ such that $\int_{a}^{\tau} r^{-1 /(\lambda+1)}(t) \Delta_{\alpha} t<\infty$. If $g:[a, \tau]_{\mathbb{T}} \rightarrow \mathbb{R}$ with $\mathrm{g}(\mathrm{a})=0$, and $\alpha \in(0,1]$, then for $\lambda \geqslant 0$

$$
\int_{a}^{\tau} s(t)|g(t)|^{\lambda}\left|T_{\alpha}^{\Delta} g(t)\right| \Delta_{\alpha} t \leqslant K_{1}(a, \tau, \lambda) \int_{a}^{\tau} r(t)\left|T_{\alpha}^{\Delta} g(t)\right|^{\lambda+1} \Delta_{\alpha} t,
$$

where

$$
K_{1}(a, \tau, \lambda)=\frac{1}{\lambda+1}\left(\int_{a}^{\tau}\left(\frac{s(t)}{r(t)}\right)^{1 / \lambda} \Delta_{\alpha} t\right)^{\lambda} .
$$

Proof. Let $u(t)=\int_{a}^{t} s^{\frac{1}{\lambda+1}}(x)\left|T_{\alpha}^{\Delta} g(x)\right| \Delta_{\alpha} x$, for $t \in[a, \tau]_{\mathbb{T}}$. Then $u(a)=0$ and

$$
\begin{aligned}
|g(t)| \leqslant \int_{a}^{t}\left|T_{\alpha}^{\Delta} g(x)\right| \Delta_{\alpha} x & \leqslant \int_{a}^{t} s^{\frac{-1}{\lambda+1}}(x) s^{\frac{1}{\lambda+1}}(x)\left|T_{\alpha}^{\Delta} g(x)\right| \Delta_{\alpha} x \\
& \leqslant s^{\frac{-1}{\lambda+1}}(t) \int_{a}^{t} s^{\frac{1}{\lambda+1}}(x)\left|T_{\alpha}^{\Delta} g(x)\right| \Delta_{\alpha} x \leqslant s^{\frac{-1}{\lambda+1}}(t) u(t) .
\end{aligned}
$$

By applying the chain rule (2.1), we obtain

$$
\mathrm{T}_{\alpha}^{\Delta}\left(\mathrm{u}^{\lambda+1}(\mathrm{t})\right)=(\lambda+1) \mathrm{u}^{\lambda}(\mathrm{d}) \mathrm{T}_{\alpha}^{\Delta} \mathrm{u}(\mathrm{t}), \text { where } \mathrm{d} \in[\mathrm{t}, \sigma(\mathrm{t})] .
$$

Since $T_{\alpha}^{\Delta} \mathfrak{u}(t)=s^{\frac{1}{\lambda+1}}(t)\left|T_{\alpha}^{\Delta} g(t)\right|>0$, and $d \geqslant t$, we see that

$$
\mathrm{T}_{\alpha}^{\Delta}\left(\mathrm{u}^{\lambda+1}(\mathrm{t})\right)=(\lambda+1) \mathrm{u}^{\lambda}(\mathrm{d}) \mathrm{T}_{\alpha}^{\Delta} \mathrm{u}(\mathrm{t}) \geqslant(\lambda+1) \mathrm{u}^{\lambda}(\mathrm{t}) \mathrm{T}_{\alpha}^{\Delta} \mathrm{u}(\mathrm{t}) .
$$

Now, from (3.34) and (3.35) we have that

$$
\begin{aligned}
\int_{a}^{\tau} s(t)|g(t)|^{\lambda}\left|T_{\alpha}^{\Delta} g(t)\right| \Delta_{\alpha} t & \leqslant \int_{a}^{\tau} s^{\frac{\lambda}{\lambda+1}}(t)|g(t)|^{\lambda} s^{\frac{1}{\lambda+1}}(t)\left|T_{\alpha}^{\Delta} g(t)\right| \Delta_{\alpha} t \\
& \leqslant \int_{a}^{\tau} u^{\lambda}(t) T_{\alpha}^{\Delta} u(t) \Delta_{\alpha} t \\
& \leqslant \int_{a}^{\tau} u^{\lambda}(d) T_{\alpha}^{\Delta} u(t) \Delta_{\alpha} t \leqslant \frac{1}{\lambda+1} \int_{a}^{\tau} T_{\alpha}^{\Delta}\left(u^{\lambda+1}(t)\right) \Delta_{\alpha} t=\frac{1}{\lambda+1} u^{\lambda+1}(\tau)
\end{aligned}
$$

By applying Hölder's inequality (2.2) with indices $(\lambda+1)$ and $(\lambda+1) / \lambda$, we see that

$$
u(\tau)=\int_{a}^{\tau} s^{\frac{1}{\lambda+1}}(t)\left|T_{\alpha}^{\Delta} g(t)\right| \Delta_{\alpha} t
$$




$$
\begin{aligned}
& =\int_{a}^{\tau} s^{\frac{1}{\lambda+1}}(t) r^{\frac{-1}{\lambda+1}}(t) r^{\frac{1}{\lambda+1}}(t)\left|T_{\alpha}^{\Delta} g(t)\right| \Delta_{\alpha} t \\
& \leqslant\left(\int_{a}^{\tau}\left(s^{\frac{1}{\lambda+1}}(t) r^{\frac{-1}{\lambda+1}}(t)\right)^{\frac{\lambda+1}{\lambda}} \Delta_{\alpha} t\right)^{\frac{\lambda}{\lambda+1}}\left(\int_{a}^{\tau}\left(r^{\frac{1}{\lambda+1}}(t)\left|T_{\alpha}^{\Delta} g(t)\right|\right)^{\lambda+1} \Delta_{\alpha} t\right)^{\frac{1}{\lambda+1}} \\
& \leqslant\left(\int_{a}^{\tau}\left(\frac{s(t)}{r(t)}\right)^{1 / \lambda} \Delta_{\alpha} t\right)^{\frac{\lambda}{\lambda+1}}\left(\int_{a}^{\tau} r(t)\left|T_{\alpha}^{\Delta} g(t)\right|^{\lambda+1} \Delta_{\alpha} t\right)^{\frac{1}{\lambda+1}} .
\end{aligned}
$$

From (3.36) and (3.37), we have

$$
\int_{a}^{\tau} s(t)|g(t)|^{\lambda}\left|T_{\alpha}^{\Delta} g(t)\right| \Delta_{\alpha} t \leqslant \frac{1}{\lambda+1} u^{\lambda+1}(\tau) \leqslant K_{1}(a, \tau, \lambda) \int_{a}^{\tau} r(t)\left|T_{\alpha}^{\Delta} g(t)\right|^{\lambda+1} \Delta_{\alpha} t
$$

where

$$
K_{1}(a, \tau, \lambda)=\frac{1}{\lambda+1}\left(\int_{a}^{\tau}\left(\frac{s(t)}{r(t)}\right)^{\frac{1}{\lambda}} \Delta_{\alpha} t\right)^{\lambda},
$$

which is the desired inequality (3.33). The proof is complete.

Remark 3.12. As a special case of Theorem 3.11, when $s(t)=r(t)$, then we get

$$
\int_{a}^{\tau} r(t)|g(t)|^{\lambda}\left|T_{\alpha}^{\Delta} g(t)\right| \Delta_{\alpha} t \leqslant\left(\frac{1}{\lambda+1}\right)\left(\frac{\tau^{\alpha}-a^{\alpha}}{\alpha}\right)^{\lambda} \int_{a}^{\tau} r(t)\left|T_{\alpha}^{\Delta} g(t)\right|^{\lambda+1} \Delta_{\alpha} t,
$$

and when $\alpha=1$, then $T_{\alpha}^{\Delta} g(t)=g^{\Delta}(t)$, and we obtain the inequality

$$
\int_{a}^{\tau} r(t)|g(t)|^{\lambda}\left|g^{\Delta}(t)\right| \Delta t \leqslant \frac{(\tau-a)^{\lambda}}{\lambda+1} \int_{a}^{\tau} r(t)\left|g^{\Delta}(t)\right|^{\lambda+1} \Delta t .
$$

Note that when $\lambda=1$, then the inequality (3.38) becomes

$$
\int_{a}^{\tau} r(t)|g(t)|\left|g^{\Delta}(t)\right| \Delta t \leqslant \frac{(\tau-a)}{2} \int_{a}^{\tau} r(t)\left|g^{\Delta}(t)\right|^{2} \Delta t
$$

Theorem 3.13. Let $\mathbb{T}$ be a time scale with $\tau, \mathrm{b} \in \mathbb{T}, \gamma>1$, and $\mathrm{s} \in \mathrm{C}_{\mathrm{rd}}\left([\tau, \mathrm{b}]_{\mathbb{T}}, \mathbb{R}^{+}\right)$be such that $\mathrm{s}(\mathrm{t})$ is nondecreasing on $[\tau, b]_{\mathbb{T}}$, and let $r$ be a nonnegative function on $(\tau, b)_{\mathbb{T}}$ such that $\int_{\tau}^{b} r^{-1 /(\lambda+\gamma)}(t) \Delta_{\alpha} t<\infty$. If $\mathrm{g}:[\tau, \mathrm{b}]_{\mathbb{T}} \rightarrow \mathbb{R}$ with $\mathrm{g}(\mathrm{b})=0$, and $\alpha \in(0,1]$, then for $\lambda \geqslant 0$

$$
\int_{\tau}^{b} s(t)\left|g^{\sigma}(t)\right|^{\lambda}\left|T_{\alpha}^{\Delta} g(t)\right|^{\gamma} \Delta_{\alpha} t \leqslant K_{2}(\tau, b, \lambda, \gamma) \int_{\tau}^{b} r(t)\left|T_{\alpha}^{\Delta} g(t)\right|^{\lambda+\gamma} \Delta_{\alpha} t,
$$

where

$$
\mathrm{K}_{2}(\tau, \mathrm{b}, \lambda, \gamma)=\left(\frac{\gamma}{\lambda+\gamma}\right)\left(\frac{\mathrm{b}^{\alpha}-\tau^{\alpha}}{\alpha}\right)^{\frac{\lambda(\gamma-1)}{\gamma}}\left(\int_{\tau}^{\mathrm{b}}\left(\frac{s(\mathrm{t})}{\mathrm{r}(\mathrm{t})}\right)^{\gamma / \lambda} \Delta_{\alpha} \mathrm{t}\right)^{\lambda / \gamma} .
$$

Proof. Let $u(t)=\int_{t}^{b} s^{\frac{\gamma}{\lambda+\gamma}}(x)\left|T_{\alpha}^{\Delta} g(x)\right|^{\gamma} \Delta_{\alpha} x$, for $t \in[\tau, b]_{\mathbb{T}}$. Then $u(b)=0$ and

$$
\mathrm{T}_{\alpha}^{\Delta} \mathrm{u}(\mathrm{t})=-\mathrm{s}^{\frac{\gamma}{\lambda+\gamma}}(\mathrm{t})\left|\mathrm{T}_{\alpha}^{\Delta} \mathrm{g}(\mathrm{t})\right|^{\gamma}<0 .
$$


Applying Hölder's inequality (2.2) with indices $\gamma$ and $\gamma /(\gamma-1)$ so that

$$
\begin{aligned}
|g(t)|=\left|\int_{t}^{b} T_{\alpha}^{\Delta} g(x) \Delta_{\alpha} x\right| & \leqslant \int_{t}^{b}\left|T_{\alpha}^{\Delta} g(x)\right| \Delta_{\alpha} x \\
& =\int_{t}^{b} s^{\frac{-1}{\lambda+\gamma}}(x) s^{\frac{1}{\lambda+\gamma}}(x)\left|T_{\alpha}^{\Delta} g(x)\right| \Delta_{\alpha} x \\
& \leqslant\left(\int_{t}^{b}\left(s^{\frac{-1}{\lambda+\gamma}}(x)\right)^{\frac{\gamma}{\gamma-1}} \Delta_{\alpha} x\right)^{\frac{\gamma-1}{\gamma}}\left(\int_{t}^{b} s^{\frac{\gamma}{\lambda+\gamma}}(x)\left|T_{\alpha}^{\Delta} g(x)\right|^{\gamma} \Delta_{\alpha} x\right)^{\frac{1}{\gamma}} \\
& \leqslant s^{\frac{-1}{\lambda+\gamma}}(t)\left(\int_{t}^{b} \Delta_{\alpha} x\right)^{\frac{\gamma-1}{\gamma}}\left(\int_{t}^{b} s^{\frac{\gamma}{\lambda+\gamma}}(x)\left|T_{\alpha}^{\Delta} g(x)\right|^{\gamma} \Delta_{\alpha} x\right)^{\frac{1}{\gamma}} .
\end{aligned}
$$

Since $T_{\alpha}^{\Delta}\left(\frac{x^{\alpha}}{\alpha}\right) \geqslant 1$, this implies that

$$
\int_{t}^{b} \Delta_{\alpha} x \leqslant \int_{t}^{b} \mathrm{~T}_{\alpha}^{\Delta}\left(\frac{x^{\alpha}}{\alpha}\right) \Delta_{\alpha} x=\left.\frac{x^{\alpha}}{\alpha}\right|_{t} ^{b}=\frac{b^{\alpha}-t^{\alpha}}{\alpha} .
$$

Substituting (3.41) into (3.40), we see that

$$
s^{\frac{\lambda}{\lambda+\gamma}}(t)|g(t)|^{\lambda} \leqslant\left(\frac{b^{\alpha}-t^{\alpha}}{\alpha}\right)^{\frac{\lambda(\gamma-1)}{\gamma}} u^{\frac{\lambda}{\gamma}}(t) .
$$

By applying the chain rule (2.1), we obtain

$$
\mathrm{T}_{\alpha}^{\Delta}\left(\mathrm{u}^{\frac{\lambda+\gamma}{\gamma}}(\mathrm{t})\right)=\frac{\lambda+\gamma}{\gamma} \mathrm{u}^{\frac{\lambda}{\gamma}}(\mathrm{d}) \mathrm{T}_{\alpha}^{\Delta} \mathrm{u}(\mathrm{t}), \quad \text { where } \mathrm{d} \in[\mathrm{t}, \sigma(\mathrm{t})] .
$$

Since $T_{\alpha}^{\Delta} \mathfrak{u}(t)=-s^{\frac{\gamma}{\lambda+\gamma}}(t)\left|T_{\alpha}^{\Delta} g(t)\right|^{\gamma}<0$, and $\sigma(t) \geqslant d$, this implies that

$$
-\mathfrak{u}^{\frac{\lambda}{\gamma}}(\sigma(t)) T_{\alpha}^{\Delta} \mathfrak{u}(t) \leqslant-\mathfrak{u}^{\frac{\lambda}{\gamma}}(d) T_{\alpha}^{\Delta} \mathfrak{u}(t) \leqslant-u^{\frac{\lambda}{\gamma}}(t) T_{\alpha}^{\Delta} \mathfrak{u}(t)
$$

and we see that

$$
-\mathrm{T}_{\alpha}^{\Delta}\left(\mathrm{u}^{\frac{\lambda+\gamma}{\gamma}}(\mathrm{t})\right)=-\frac{\lambda+\gamma}{\gamma} \mathrm{u}^{\frac{\lambda}{\gamma}}(\mathrm{d}) \mathrm{T}_{\alpha}^{\Delta} \mathrm{u}(\mathrm{t}) \geqslant-\frac{\lambda+\gamma}{\gamma} \mathrm{u}^{\frac{\lambda}{\gamma}}(\sigma(\mathrm{t})) \mathrm{T}_{\alpha}^{\Delta} \mathrm{u}(\mathrm{t}) .
$$

Now, from (3.42) and (3.43) we have that

$$
\begin{aligned}
\int_{\tau}^{\mathrm{b}} s(\mathrm{t})|\mathrm{g}(\sigma(\mathrm{t}))|^{\lambda}\left|\mathrm{T}_{\alpha}^{\Delta} \mathrm{g}(\mathrm{t})\right|^{\gamma} \Delta_{\alpha} \mathrm{t} & =\int_{\tau}^{\mathrm{b}} \mathrm{s}^{\frac{\lambda}{\lambda+\gamma}}(\mathrm{t})|\mathrm{g}(\sigma(\mathrm{t}))|^{\lambda} \mathrm{s}^{\frac{\gamma}{\lambda+\gamma}}(\mathrm{t})\left|\mathrm{T}_{\alpha}^{\Delta} \mathrm{g}(\mathrm{t})\right|^{\gamma} \Delta_{\alpha} \mathrm{t} \\
& \leqslant \int_{\tau}^{\mathrm{b}} \mathrm{s}^{\frac{\lambda}{\lambda+\gamma}}(\sigma(\mathrm{t}))|\mathrm{g}(\sigma(\mathrm{t}))|^{\lambda} \mathrm{s}^{\frac{\gamma}{\lambda+\gamma}}(\mathrm{t})\left|\mathrm{T}_{\alpha}^{\Delta} \mathrm{g}(\mathrm{t})\right|^{\gamma} \Delta_{\alpha} \mathrm{t} \\
& \leqslant \int_{\tau}^{\mathrm{b}}\left(\frac{\mathrm{b}^{\alpha}-(\sigma(\mathrm{t}))^{\alpha}}{\alpha}\right)^{\frac{\lambda(\gamma-1)}{\gamma}}\left(-\mathrm{u}^{\frac{\lambda}{\gamma}}(\sigma(\mathrm{t})) \mathrm{T}_{\alpha}^{\Delta} \mathrm{u}(\mathrm{t})\right) \Delta_{\alpha} \mathrm{t} \\
& \leqslant\left(\frac{\mathrm{b}^{\alpha}-\tau^{\alpha}}{\alpha}\right)^{\frac{\lambda(\gamma-1)}{\gamma}} \int_{\tau}^{\mathrm{b}}\left(-\mathrm{u}^{\frac{\lambda}{\gamma}}(\sigma(t)) \mathrm{T}_{\alpha}^{\Delta} \mathfrak{u}(\mathrm{t})\right) \Delta_{\alpha} \mathrm{t}
\end{aligned}
$$




$$
\begin{aligned}
& \leqslant \frac{\gamma}{\lambda+\gamma}\left(\frac{b^{\alpha}-\tau^{\alpha}}{\alpha}\right)^{\frac{\lambda(\gamma-1)}{\gamma}} \int_{\tau}^{b} T_{\alpha}^{\Delta}\left(-u^{\frac{\lambda+\gamma}{\gamma}}(t)\right) \Delta_{\alpha} t \\
& =\frac{\gamma}{\lambda+\gamma}\left(\frac{b^{\alpha}-\tau^{\alpha}}{\alpha}\right)^{\frac{\lambda(\gamma-1)}{\gamma}} u^{\frac{\lambda+\gamma}{\gamma}}(\tau) .
\end{aligned}
$$

By applying Hölder's inequality (2.2) with indices $(\lambda+\gamma) / \lambda$ and $(\lambda+\gamma) / \gamma$, and (3.41), we see that

$$
\begin{aligned}
\int_{\tau}^{b} s(t)|g(\sigma(t))|^{\lambda}\left|T_{\alpha}^{\Delta} g(t)\right|^{\gamma} \Delta_{\alpha} t= & \frac{\gamma}{\lambda+\gamma}\left(\frac{b^{\alpha}-\tau^{\alpha}}{\alpha}\right)^{\frac{\lambda(\gamma-1)}{\gamma}}\left(\int_{\tau}^{b} s^{\frac{\gamma}{\lambda+\gamma}}(t)\left|T_{\alpha}^{\Delta} g(t)\right|^{\gamma} \Delta_{\alpha} t\right)^{\frac{\lambda+\gamma}{\gamma}} \\
\leqslant & \frac{\gamma}{\lambda+\gamma}\left(\frac{b^{\alpha}-\tau^{\alpha}}{\alpha}\right)^{\frac{\lambda(\gamma-1)}{\gamma}}\left(\int_{\tau}^{b} s^{\frac{\gamma}{\lambda+\gamma}}(t) r^{\frac{-\gamma}{\lambda+\gamma}}(t)\right. \\
& \left.\times r^{\frac{\gamma}{\lambda+\gamma}}(t)\left|T_{\alpha}^{\Delta} g(t)\right|^{\gamma} \Delta_{\alpha} t\right)^{\frac{\lambda+\gamma}{\gamma}} \\
\leqslant & \frac{\gamma}{\lambda+\gamma}\left(\frac{b^{\alpha}-\tau^{\alpha}}{\alpha}\right)^{\frac{\lambda(\gamma-1)}{\gamma}}\left(\int_{\tau}^{b}\left(\frac{s(t)}{r(t)}\right)^{\gamma / \lambda} \Delta_{\alpha} t\right)^{\lambda / \gamma} \\
& \times \int_{\tau}^{b} r(t)\left|T_{\alpha}^{\Delta} g(t)\right|^{\lambda+\gamma} \Delta_{\alpha} t \\
= & K_{2}(\tau, b, \lambda, \gamma) \int_{\tau}^{b} r(t)\left|T_{\alpha}^{\Delta} g(t)\right|^{\lambda+\gamma} \Delta_{\alpha} t,
\end{aligned}
$$

where

$$
\mathrm{K}_{2}(\tau, \mathrm{b}, \lambda, \gamma)=\frac{\gamma}{\lambda+\gamma}\left(\frac{\mathrm{b}^{\alpha}-\tau^{\alpha}}{\alpha}\right)^{\frac{\lambda(\gamma-1)}{\gamma}}\left(\int_{\tau}^{\mathrm{b}}\left(\frac{\mathrm{s}(\mathrm{t})}{\mathrm{r}(\mathrm{t})}\right)^{\gamma / \lambda} \Delta_{\alpha} \mathrm{t}\right)^{\lambda / \gamma},
$$

which is the desired inequality (3.39). The proof is complete.

Remark 3.14. As a special case of Theorem 3.13, when $s(t)=r(t)$, then we get

$$
\int_{\tau}^{b} r(t)\left|g^{\sigma}(t)\right|^{\lambda}\left|T_{\alpha}^{\Delta} g(t)\right|^{\gamma} \Delta_{\alpha} t \leqslant \frac{\gamma}{\lambda+\gamma}\left(\frac{b^{\alpha}-\tau^{\alpha}}{\alpha}\right)^{\lambda} \int_{\tau}^{b} r(t)\left|T_{\alpha}^{\Delta} g(t)\right|^{\lambda+\gamma} \Delta_{\alpha} t,
$$

and when $\alpha=1$, then $T_{\alpha}^{\Delta} g(t)=g^{\Delta}(t)$, and we obtain the inequality

$$
\int_{\tau}^{b} r(t)\left|g^{\sigma}(t)\right|^{\lambda}\left|g^{\Delta}(t)\right|^{\gamma} \Delta t \leqslant \frac{\gamma(b-\tau)^{\lambda}}{\lambda+\gamma} \int_{\tau}^{b} r(t)\left|g^{\Delta}(t)\right|^{\lambda+\gamma} \Delta t .
$$

Theorem 3.15. Let $\mathbb{T}$ be a time scale with $\tau, \mathrm{b} \in \mathbb{T}$, and $\mathrm{s} \in \mathrm{C}_{\mathrm{rd}}\left([\tau, \mathrm{b}]_{\mathbb{T}}, \mathbb{R}^{+}\right)$be such that $\mathrm{s}(\mathrm{t})$ is nondecreasing on $[\tau, \mathrm{b}]_{\mathbb{T}}$, and let $\mathrm{r}$ be a nonnegative function on $(\tau, \mathrm{b})_{\mathbb{T}}$ such that $\int_{\tau}^{\mathrm{b}} \mathrm{r}^{-1 /(\lambda+\gamma)}(\mathrm{t}) \Delta_{\alpha} \mathrm{t}<\infty$. If $\mathrm{g}:[\tau, \mathrm{b}]_{\mathbb{T}} \rightarrow \mathbb{R}$ with $\mathrm{g}(\mathrm{b})=0$, and $\alpha \in(0,1]$, then for $\lambda \geqslant 0$

$$
\int_{\tau}^{b} s(t)\left|g^{\sigma}(t)\right|^{\lambda}\left|T_{\alpha}^{\Delta} g(t)\right| \Delta_{\alpha} t \leqslant K_{2}(\tau, b, \lambda) \int_{\tau}^{b} r(t)\left|T_{\alpha}^{\Delta} g(t)\right|^{\lambda+1} \Delta_{\alpha} t
$$

where

$$
K_{2}(\tau, b, \lambda)=\frac{1}{\lambda+1}\left(\int_{\tau}^{b}\left(\frac{s(t)}{r(t)}\right)^{\frac{1}{\lambda}} \Delta_{\alpha} t\right)^{\lambda}
$$


Proof. Let $u(t)=\int_{t}^{b} s^{\frac{1}{\lambda+1}}(x)\left|T_{\alpha}^{\Delta} g(x)\right| \Delta_{\alpha} x$, for $t \in[\tau, b]_{\mathbb{T}}$. Then $u(b)=0$ and

$$
\begin{aligned}
|g(t)|=\left|\int_{t}^{b} T_{\alpha}^{\Delta} g(x) \Delta_{\alpha} x\right| & \leqslant \int_{t}^{b}\left|T_{\alpha}^{\Delta} g(x)\right| \Delta_{\alpha} x \\
& =\int_{t}^{b} s^{\frac{-1}{\lambda+1}}(x) s^{\frac{1}{\lambda+1}}(x)\left|T_{\alpha}^{\Delta} g(x)\right| \Delta_{\alpha} x \\
& \leqslant s^{\frac{-1}{\lambda+1}}(t) \int_{t}^{b} s^{\frac{1}{\lambda+1}}(x)\left|T_{\alpha}^{\Delta} g(x)\right| \Delta_{\alpha} x \leqslant s^{\frac{-1}{\lambda+1}}(t) u(t) .
\end{aligned}
$$

By applying the chain rule (2.1), we obtain

$$
\mathrm{T}_{\alpha}^{\Delta} \mathrm{u}^{\lambda+1}(\mathrm{t})=(\lambda+1) \mathrm{u}^{\lambda}(\mathrm{d}) \mathrm{T}_{\alpha}^{\Delta} \mathfrak{u}(\mathrm{t}), \text { where } \mathrm{d} \in[\mathrm{t}, \sigma(\mathrm{t})] .
$$

Since $T_{\alpha}^{\Delta} u(t)=-s^{\frac{1}{\lambda+1}}(t)\left|T_{\alpha}^{\Delta} g(t)\right|<0$, and $\sigma(t) \geqslant d$, this implies that

$$
-u^{\lambda}(\sigma(t)) T_{\alpha}^{\Delta} u(t) \leqslant-u^{\lambda}(d) T_{\alpha}^{\Delta} u(t) \leqslant-u^{\lambda}(t) T_{\alpha}^{\Delta} u(t)
$$

and we see that

$$
-T_{\alpha}^{\Delta} u^{\lambda+1}(t)=-(\lambda+1) u^{\lambda}(d) T_{\alpha}^{\Delta} u(t) \geqslant-(\lambda+1) u^{\lambda}(\sigma(t)) T_{\alpha}^{\Delta} u(t) .
$$

Now, from (3.45) and (3.46) we have that

$$
\begin{aligned}
\int_{\tau}^{b} s(t)\left|g^{\sigma}(t)\right|^{\lambda}\left|T_{\alpha}^{\Delta} g(t)\right| \Delta_{\alpha} t & =\int_{\tau}^{b} s^{\frac{\lambda}{\lambda+1}}(t)\left|g^{\sigma}(t)\right|^{\lambda} s^{\frac{1}{\lambda+1}}(t)\left|T_{\alpha}^{\Delta} g(t)\right| \Delta_{\alpha} t \\
& \leqslant \int_{\tau}^{b} s^{\frac{\lambda}{\lambda+1}}(\sigma(t))\left|g^{\sigma}(t)\right|^{\lambda} s^{\frac{1}{\lambda+1}}(t)\left|T_{\alpha}^{\Delta} g(t)\right| \Delta_{\alpha} t \\
& \leqslant \int_{\tau}^{b}\left(-u^{\lambda}(\sigma(t)) T_{\alpha}^{\Delta} u(t)\right) \Delta_{\alpha} t \leqslant \int_{\tau}^{b}\left(-u^{\lambda}(d) T_{\alpha}^{\Delta} u(t)\right) \Delta_{\alpha} t \\
& =\frac{1}{\lambda+1} \int_{\tau}^{b}\left(-T_{\alpha}^{\Delta} u^{\lambda+1}(t)\right) \Delta_{\alpha} t=\frac{1}{\lambda+1} u^{\lambda+1}(\tau) .
\end{aligned}
$$

By applying Hölder's inequality (2.2) with indices $(\lambda+1)$ and $(\lambda+1) / \lambda$, we have

$$
\begin{aligned}
\int_{\tau}^{\mathrm{b}} \mathrm{s}(\mathrm{t})\left|\mathrm{g}^{\sigma}(\mathrm{t})\right|^{\lambda}\left|\mathrm{T}_{\alpha}^{\Delta} \mathrm{g}(\mathrm{t})\right| \Delta_{\alpha} \mathrm{t} & \leqslant \frac{1}{\lambda+1} \mathrm{u}^{\lambda+1}(\tau) \\
& =\frac{1}{\lambda+1}\left(\int_{\tau}^{\mathrm{b}} \mathrm{s}^{\frac{1}{\lambda+1}}(\mathrm{t})\left|\mathrm{T}_{\alpha}^{\Delta} \mathrm{g}(\mathrm{t})\right| \Delta_{\alpha} \mathrm{t}\right)^{\lambda+1} \\
& =\frac{1}{\lambda+1}\left(\int_{\tau}^{\mathrm{b}} \mathrm{s}^{\frac{1}{\lambda+1}}(\mathrm{t}) \mathrm{r}^{\frac{-1}{\lambda+1}}(\mathrm{t}) \mathrm{r}^{\frac{1}{\lambda+1}}(\mathrm{t})\left|\mathrm{T}_{\alpha}^{\Delta} \mathrm{g}(\mathrm{t})\right| \Delta_{\alpha} \mathrm{t}\right)^{\lambda+1} \\
& =\frac{1}{\lambda+1}\left(\int_{\tau}^{\mathrm{b}}\left(\frac{\mathrm{s}(\mathrm{t})}{\mathrm{r}(\mathrm{t})}\right)^{\frac{1}{\lambda+1}} \mathrm{r}^{\frac{1}{\lambda+1}}(\mathrm{t})\left|\mathrm{T}_{\alpha}^{\Delta} \mathrm{g}(\mathrm{t})\right| \Delta_{\alpha} \mathrm{t}\right)^{\lambda+1}
\end{aligned}
$$




$$
\begin{aligned}
& \leqslant \frac{1}{\lambda+1}\left(\int_{\tau}^{\mathrm{b}}\left(\frac{\mathrm{s}(\mathrm{t})}{\mathrm{r}(\mathrm{t})}\right)^{\frac{1}{\lambda}} \Delta_{\alpha} \mathrm{t}\right)^{\lambda} \int_{\tau}^{\mathrm{b}} r(\mathrm{t})\left|\mathrm{T}_{\alpha}^{\Delta} \mathrm{g}(\mathrm{t})\right|^{\lambda+1} \Delta_{\alpha} \mathrm{t} \\
& =\mathrm{K}_{2}(\tau, \mathrm{b}, \lambda) \int_{\tau}^{\mathrm{b}} \mathrm{r}(\mathrm{t})\left|\mathrm{T}_{\alpha}^{\Delta} \mathrm{g}(\mathrm{t})\right|^{\lambda+1} \Delta_{\alpha} \mathrm{t},
\end{aligned}
$$

where

$$
K_{2}(\tau, b, \lambda)=\frac{1}{\lambda+1}\left(\int_{\tau}^{b}\left(\frac{s(t)}{r(t)}\right)^{\frac{1}{\lambda}} \Delta_{\alpha} t\right)^{\lambda},
$$

which is the desired inequality (3.44). The proof is complete.

Remark 3.16. As a special case of Theorem 3.15, when $s(t)=r(t)$, then we get

$$
\int_{\tau}^{b} r(t)\left|g^{\sigma}(t)\right|^{\lambda}\left|T_{\alpha}^{\Delta} g(t)\right| \Delta_{\alpha} t \leqslant \frac{1}{\lambda+1}\left(\frac{b^{\alpha}-\tau^{\alpha}}{\alpha}\right)^{\lambda} \int_{\tau}^{b} r(t)\left|T_{\alpha}^{\Delta} g(t)\right|^{\lambda+1} \Delta_{\alpha} t
$$

and when $\alpha=1$, then $T_{\alpha}^{\Delta} g(t)=g^{\Delta}(t)$, and we obtain the inequality

$$
\int_{\tau}^{b} r(t)\left|g^{\sigma}(t)\right|^{\lambda}\left|g^{\Delta}(t)\right| \Delta t \leqslant \frac{(b-\tau)^{\lambda}}{\lambda+1} \int_{\tau}^{b} r(t)\left|g^{\Delta}(t)\right|^{\lambda+1} \Delta t
$$

Note that when $\lambda=1$, then the inequality (3.47) becomes

$$
\int_{\tau}^{b} r(t)\left|g^{\sigma}(t)\right|\left|g^{\Delta}(t)\right| \Delta t \leqslant \frac{(b-\tau)}{2} \int_{\tau}^{b} r(t)\left|g^{\Delta}(t)\right|^{2} \Delta t .
$$

\section{References}

[1] T. Abdeljawad, On conformable fractional calculus, J. Comput. Appl. Math., 279 (2015), 57-66. 1

[2] R. P. Agarwal, M. Bohner, Basic calculus on time scales and some of its applications, Results Math., 35 (1999), 3-22.

[3] R. P. Agarwal, D. O’Regan, S. H. Saker, Dynamic Inequalities on Time Scales, Springer, Cham, (2014).

[4] A. M. Ahmed, G. AlNemer, M. Zakarya, H. M. Rezk, Some Dynamic Inequalities of Hilbert's Type, J. Funct. Spaces, 2020 (2020), 13 pages.

[5] A. Akkurt, M. E. Yildirim, H. Yildirim, On some integral inequalities for conformable fractional integrals, Asian J. Math. Comput. Res., 15 (2017), 205-212. 1

[6] N. Benkhettou, S. Hassani, D. F. M. Torres, A conformable fractional calculus on arbitrary time scales, J. King Saud Univ. Sci., 28 (2016), 93-98. 2

[7] K. Bogdan, B. Dyda, The best constant in a fractional Hardy inequality, Math. Nachr., 284 (2011), 629-638. 1

[8] M. Bohner, T. S. Hassan, T. X. Li, Fite-Hille-Wintner-type oscillation criteria for second-order half-linear dynamic equation with deviating arguments, Indag. Math. (N.S.), 29 (2018), 548-560.

[9] M. Bohner, B. KaymakÇalan, Opial inequalities on time scales, Ann. Polon. Math., 77 (2001), 11-20. 1, 1

[10] M. Bohner, T. X. Li, Kamenev-type criteria for nonlinear damped dynamic equations, Sci. China Math., 58 (2015), 14451452.

[11] M. Bohner, A. Peterson, Dynamic Equations on Time Scales: An Introduction with Applications, Birkhäuser, Boston, (2001). 2

[12] M. Bohner, A. Peterson, Advances in Dynamic Equations on Time Scales, Birkhäuser Boston, Boston, (2003). 2

[13] Y.-M. Chu, M. A. Khan, T. Ali, S. S. Dragomir, Inequalities for $\alpha$-fractional differentiable functions, J. Inequal. Appl., 2017 (2017), 12 pages. 1

[14] S. Hilger, Analysis on measure chains a unified approach to continuous and discrete calculus, Results Math., 18 (1990), 18-56.

[15] M. Jleli, B. Samet, Lyapunov-type inequalities for a fractional differential equation with mixed boundary conditions, Math. Inequal. Appl., 18 (2015), 443-451. 1

[16] V. Kac, P. Cheung, Quantum Calculus, Springer-Verlag, New York, (2001). 
[17] B. Karpuz, B. KaymakÇalan, Ö. Öclan, A generalization of Opial's inequality and applications to second order dynamic equations, Differ. Equ. Dyn. Syst., 18 (2010), 11-18. 1

[18] R. Khalil, M. Al Horani, A. Yousef, M. Sababheh, A new definition of fractional derivative, J. Comput. Appl. Math., 264 (2014), 65-70. 1

[19] M. A. Khan, T. Ali, S. S. Dragomir, M. Z. Sarikaya, Hermite-Hadamard type inequalities for conformable fractional integrals, Rev. R. Acad. Cienc. Exactas Fis. Nat. Ser. A Mat. RACSAM, 112 (2018), 1033-1048. 1

[20] F. J. Martin-Reyes, E. Sawyer, Weighted inequalities for Riemann-Liouville fractional integrals of order one and greater, Proc. Amer. Math. Soc., 106 (1989), 727-733.

[21] E. R. Nwaeze, D. F. M Torres, Chain rules and inequalities for the BHT fractional calculus on arbitrary timescales, Arab. J. Math. (Springer), 6 (2017), 13-20. 2

[22] B. G. Pachpatte, On Opial-type integral inequalities, J. Math. Anal. Appl., 120 (1986), 547-556.

[23] S. H. Saker, Some nonlinear dynamic inequalities on time scales and applications, J. Math. Inequal.,4 (2010), 561-579.

[24] S. H. Saker, Some Opial-type inequalities on time scales, Abstr. Appl. Anal., 2011 (2011), 19 pages. 1, 1

[25] S. H. Saker, New inequalities of Opial's type on time scales and some of their applications, Discrete Dyn. Nat. Soc., 2012 (2012), 23 pages.

[26] S. H. Saker, M. R. Kenawy, G. AlNemer, M. Zakarya, Some Fractional Dynamic Inequalities of Hardy'sType Via Conformable Calculus, Mathematics, 8 (2020), 15 pages.

[27] M. Z. Sarikaya, H. Budak, New inequalities of Opial type for conformable fractional integrals, Turkish J. Math., 41 (2017), 1164-1173. 1

[28] M. Z. Sarikaya, H. Budak, Opial type inequalities for conformable fractional integrals, J. Appl. Anal., 25 (2019), $155-163$. 1

[29] M. Z. Sarikaya, H. Yaldiz, H. Budak, Steffensen's integral inequality for conformable fractional integrals, Int. J. Anal. Appl., 15 (2017), 23-30. 1

[30] E. Set, A. Gözpınar, A. Ekinci, Hermite-Hadamard type inequalities via conformable fractional integrals, Acta Math. Univ. Comenian. (N.S.), 86 (2017), 309-320.

[31] H. M. Srivastava, K.-L. Tseng, S.-J. Tseng, J.-C. Lo, Some weighted Opial-type inequalities on time scale, Taiwanese J. Math., 14 (2010), 107-122. 1

[32] F. Usta, M. Z. Sarikaya, Some improvements of conformable fractional integral inequalities, Int. J. Anal. Appl., 14 (2017), 162-166.

[33] F.-H. Wong, W.-C. Lin, S.-L. Yu, C.-C. Yeh, Some generalizations of Opial's inequalities on time scales, Taiwanese J. Math., 12 (2008), 463-471. 1

[34] C. Yildiz, M. E. Ozdemir, H. Kavurmac1Onelan, Fractional integral inequalities for different functions, New Trends Math. Sci., 3 (2015), 110-117. 1 